\title{
TRAVELING WAVES IN A SIRH MODEL WITH SPATIO-TEMPORAL DELAY AND NONLOCAL DISPERSAL*
}

\author{
Lu YANG (杨路) Yun-Rui YANG (杨羿瑞) ${ }^{\dagger} \quad$ Xue SONG (宋雪) \\ School of Mathematics and Physics, Lanzhou Jiaotong University, Lanzhou 730070, China \\ E-mail: yanglu19970729@163.com; lily1979101@163.com; sx18604126839@163.com
}

\begin{abstract}
This paper deals mainly with the existence and asymptotic behavior of traveling waves in a SIRH model with spatio-temporal delay and nonlocal dispersal based on Schauder's fixed-point theorem and analysis techniques, which generalize the results of nonlocal SIRH models without relapse and delay. In particular, the difficulty of obtaining the asymptotic behavior of traveling waves for the appearance of spatio-temporal delay is overcome by the use of integral techniques and analysis techniques. Finally, the more general nonexistence result of traveling waves is also included.
\end{abstract}

Key words Nonlocal dispersal; spatio-temporal delay; traveling waves

2010 MR Subject Classification 35K57; 92D25; 35A01

\section{Introduction}

Traveling waves of reaction-diffusion equations have long been the subject of investigation, due to the fact that many propagation phenomena in nature can be described by them, including population diffusion, species invasion, disease transmission and so on [1-3]. Generally, the equations with Laplace (local) diffusion $[4,5]$ operate on the basis that the dispersal of individuals is only affected by diffusion at single point. In actual fact, the dispersal of individuals depends not only on one point, but on others around it, even farther away it, and this is what is referred to as nonlocal dispersal [6,7]. This kind of dispersal is expressed by

$$
J * u(x, t)-u(x, t)=\int_{R} J(x-y)(u(y, t)-u(x, t)) \mathrm{d} y,
$$

and is used to illustrate long-range diffusion, which means, in biology, the net growth rate of $u(x, t)$, where $u(x, t)$ represents the population density of some species at position $x$ and time $t$. Based on this, much attention has been paid to traveling waves of nonlocal dispersal models, especially for epidemic models with nonlocal dispersal [8-12]. For example, Li and Yang [9]

${ }^{*}$ Received October 16, 2020; revised January 4, 2021. Yun-Rui Yang is supported by the NSF of China (11761046), Science and Technology Plan Foundation of Gansu Province of China (20JR5RA411), and Foundation of A Hundred Youth Talents Training Program of Lanzhou Jiaotong University.

${ }^{\dagger}$ Corresponding author 
considered the (non)existence of traveling waves for the SIR model

$$
\left\{\begin{array}{l}
\frac{\partial U(x, t)}{\partial t}=d_{1}(J * U(x, t)-U(x, t))-\frac{\alpha U(x, t) V(x, t)}{U(x, t)+V(x, t)} \\
\frac{\partial V(x, t)}{\partial t}=d_{2}(J * V(x, t)-V(x, t))+\frac{\alpha U(x, t) V(x, t)}{U(x, t)+V(x, t)}-\beta V \\
\frac{\partial W(x, t)}{\partial t}=d_{3}(J * W(x, t)-W(x, t))+\beta V
\end{array}\right.
$$

with nonlocal dispersal, where $U, V$ and $W$ are the densities of susceptible, infected, and cured individuals, respectively. $d_{i}>0(i=1,2,3)$ indicates the dispersal rate, $\alpha$ represents the infection rate, and $\beta$ denotes the recovery (treatment) rate.

On the other hand, time delay is actually inevitable for many phenomena in the real world, such as the latency period of bacteria, digestion periods, maturation periods of species and so on. Consequently, traveling waves of those models with nonlocal dispersal and delay have been extensively investigated [13-15]. In 2020, Yang et al. [15] established the (non)existence, boundedness and asymptotic behavior of traveling waves for the nonlocal dispersal SIR model

$$
\left\{\begin{array}{l}
\frac{\partial U(x, t)}{\partial t}=d_{1}(J * U(x, t)-U(x, t))-f(U(x, t)) g(V(x, t-\tau)), \\
\frac{\partial V(x, t)}{\partial t}=d_{2}(J * V(x, t)-V(x, t))-f(U(x, t)) g(V(x, t-\tau))-\beta V(x, t), \\
\frac{\partial W(x, t)}{\partial t}=d_{3}(J * W(x, t)-W(x, t))+\beta V(x, t),
\end{array}\right.
$$

equipped with delay and nonlinear incidence. In addition, in view of the fact that every possible location of an individual in all previous times has an impact on the current state, Britton $[16,17]$ and Smith [18] introduced what they called "time and space nonlocality"; namely, spatiotemporal delay by the idea of a spatial weighted average and a method of characteristic lines. Since then, the investigation of traveling waves for nonlocal dispersal equations with spatiotemporal delay has made great progress [19-24]. For example, by Schauder's fixed theorem, together with a two-sided Laplace transform, Wang et al. [19] extended the (non)existence results for traveling waves of (1.1) to the model

$$
\left\{\begin{array}{l}
\frac{\partial U(x, t)}{\partial t}=d_{1}\left(J_{\gamma_{1}} * U(x, t)-U(x, t)\right)-\frac{\alpha U(x, t) G * V(x, t)}{U(x, t)+G * V(x, t)}, \\
\frac{\partial V(x, t)}{\partial t}=d_{2}\left(J_{\gamma_{2}} * V(x, t)-V(x, t)\right)+\frac{\alpha U(x, t) G * V(x, t)}{U(x, t)+G * V(x, t)}-\beta V(x, t), \\
\frac{\partial W(x, t)}{\partial t}=d_{3}\left(J_{\gamma_{3}} * W(x, t)-W(x, t)\right)+\beta V(x, t)
\end{array}\right.
$$

with spatio-temporal delay, where $G * V(x, t)=\int_{0}^{T} \int_{-\infty}^{+\infty} G(y, s) V(x-y, t-s) \mathrm{d} y \mathrm{~d} s$ and $0<$ $T<+\infty$ is the longest latent period during which a susceptible becomes a infected, where $T$ may be $+\infty$ in general.

However, the above SIR models do not consider whether individuals who received treatment will be ill again or not, and therefore they are not realistic for describing infectious diseases with relapse, such as the flu, herpes, COVID-19 and so on. In 2017, Zhu et al. [12] put their 
emphasis on the SIRH model

$$
\left\{\begin{aligned}
\frac{\partial U(x, t)}{\partial t}= & d(J * U(x, t)-U(x, t))+\Lambda-\mu U(x, t)-\frac{\alpha U(x, t) V(x, t)}{U(x, t)+V(x, t)+W(x, t)}, \\
\frac{\partial V(x, t)}{\partial t}= & d(J * V(x, t)-V(x, t))+\frac{\alpha U(x, t) V(x, t)}{U(x, t)+V(x, t)+W(x, t)}+\gamma W(x, t) \\
& -\left(\mu+\eta_{1}+\beta\right) V(x, t), \\
\frac{\partial W(x, t)}{\partial t}= & d(J * W(x, t)-W(x, t))+\beta V(x, t)-\gamma W(x, t)-\left(\mu+\eta_{2}+\rho\right) W(x, t), \\
\frac{\partial H(x, t)}{\partial t}= & \rho W(x, t)-\mu H(x, t)
\end{aligned}\right.
$$

with relapse, where $\Lambda$ is the external import rate, $\mu$ represents the death rate, $\gamma$ denotes the rate of relapse, $\rho$ indicates the permanent immunity rate, and $H(x, t)$ is the density of individuals who received treatment and will never relapse again. They established the (non)existence, boundedness and asymptotic behavior of the traveling waves of (1.4) by Schauder's fixed-point theorem and analysis techniques. Notice that the effect of delay (that is, the latency period of bacteria) is not considered in the model (1.4).

As a result, based on the above works [11, 19, 22, 24] and motivated by the ideas of Wang [19] and Yang [24], we intend to generalize the above traveling wave results for (1.4) to the model

$$
\left\{\begin{aligned}
\frac{\partial U(x, t)}{\partial t}= & d(J * U(x, t)-U(x, t))+\Lambda-\mu U(x, t)-\frac{\alpha U(x, t) G * V(x, t)}{U(x, t)+G * V(x, t)+W(x, t)} \\
\frac{\partial V(x, t)}{\partial t}= & d(J * V(x, t)-V(x, t))+\frac{\alpha U(x, t) G * V(x, t)}{U(x, t)+G * V(x, t)+W(x, t)}+\gamma W(x, t) \\
& -\left(\mu+\eta_{1}+\beta\right) V(x, t), \\
\frac{\partial W(x, t)}{\partial t}= & d(J * W(x, t)-W(x, t))+\beta V(x, t)-\gamma W(x, t)-\left(\mu+\eta_{2}+\rho\right) W(x, t), \\
\frac{\partial H(x, t)}{\partial t}= & \rho W(x, t)-\mu H(x, t),
\end{aligned}\right.
$$

with relapse and spatio-temporal delay. First, we will find a positive constant $c^{*}$, which will be defined later (Lemma 2.1), to construct the existence and asymptotic behavior of the positive bounded traveling waves of (1.5) for $c>c^{*}$ by Schauder's fixed-point theorem, analysis techniques and integral techniques. It should be pointed out that we need the same diffusive rates and natural morality rate for $U, V$ and $W$ to guarantee the boundedness of traveling waves. There is a certain difficulty in establishing the asymptotic behavior of traveling waves for (1.5) with $c>c^{*}$ by the previous method in [12] for the appearance of spatio-temporal delay. Inspired by the work of Yang et al. [24], this difficulty is overcome by the use of integral techniques and analysis techniques. In addition, the existence of non-negative bounded traveling waves with $c=c^{*}$ is also obtained by the limit theory. In particular, motivated by the ideas of Yang et al. in [11], the existence and asymptotic behavior of positive bounded traveling waves of (1.5) when $c=c^{*}$ and $\gamma=0$ are proven by some analysis techniques, and this improves upon the work of Wang et al. [19] and Zhu et al. [12]. Finally, we also find another positive constant $c_{*}$, defined in Lemma 2.1, to establish the nonexistence of traveling waves of (1.5) with $0<c<c_{*}$ 
by a two-sided Laplace transform, which is different from the method of Yang et al. [24], who only obtained that there is no traveling wave solution decaying exponentially at $\xi \rightarrow-\infty$, we obtain more general nonexistence results.

It is worth noting that the two positive constants mentioned above, $c_{*}$ and $c^{*}$, satisfy $0<c_{*} \leq c^{*}$; this is attributed to the fact that the rate of relapse and spatio-temporal delay arise at the same time in the model (1.5). It is still an open problem as to whether or not there is a traveling wave when $c_{*} \leq c<c^{*}$ for $c_{*}<c^{*}$. Moreover, when the relapse and the healing individuals $H(x, t)$ are not considered, and taking $\Lambda=\mu=\gamma=H=\eta_{1}=\eta_{2}=0$, the model (1.5) becomes a SIR model with spatio-temporal delay in [22]; when $G(x, t)=\delta(x) \cdot \delta(t)$, the model (1.5) reduces to (1.4), where $\delta(\cdot)$ is Dirac function. Therefore, this paper generalizes the conclusions in $[12,19,22]$.

The rest of this article is arranged as follows: in Section 2, some of the assumptions and preliminary results we need are illustrated. In Section 3, the existence, boundedness and asymptotic behavior of traveling waves for the wave speed $c \geq c^{*}$ are established. In Section 4 , the non-existence of traveling wave solutions for $0<c<c_{*}$ is investigated.

\section{Preliminaries}

Considering the relative independence of the fourth equation in (1.5), it is sufficient to focus on the following system:

$$
\left\{\begin{aligned}
\frac{\partial U(x, t)}{\partial t}= & d(J * U(x, t)-U(x, t))+\Lambda-\mu U(x, t)-\frac{\alpha U(x, t) G * V(x, t)}{U(x, t)+G * V(x, t)+W(x, t)} \\
\frac{\partial V(x, t)}{\partial t}= & d(J * V(x, t)-V(x, t))+\frac{\alpha U(x, t) G * V(x, t)}{U(x, t)+G * V(x, t)+W(x, t)}+\gamma W(x, t) \\
& -\left(\mu+\eta_{1}+\beta\right) V(x, t), \\
\frac{\partial W(x, t)}{\partial t}= & d(J * W(x, t)-W(x, t))+\beta V(x, t)-\gamma W(x, t)-\left(\mu+\eta_{2}+\rho\right) W(x, t) .
\end{aligned}\right.
$$

Denote the initial disease-free equilibrium as $\mathbf{E}:=\left(U_{0}, 0,0\right)$. Let $(U(x, t), V(x, t), W(x, t))=$ $(U(\xi), V(\xi), W(\xi))$ and $\xi=x+c t$. Then $(U(\xi), V(\xi), W(\xi))$ satisfies the corresponding traveling wave system of $(2.1)$ as follows:

$$
\left\{\begin{array}{l}
c U^{\prime}(\xi)=d(J * U(\xi)-U(\xi))+\Lambda-\mu U(\xi)-\frac{\alpha U(\xi) G * V(\xi)}{U(\xi)+G * V(\xi)+W(\xi)}, \\
c V^{\prime}(\xi)=d(J * V(\xi)-V(\xi))+\frac{\alpha U(\xi) G * V(\xi)}{U(\xi)+G * V(\xi)+W(\xi)}+\gamma W(\xi)-\left(\mu+\eta_{1}+\beta\right) V(\xi), \\
c W^{\prime}(\xi)=d(J * W(\xi)-W(\xi))+\beta V(\xi)-\gamma W(\xi)-\left(\mu+\eta_{2}+\rho\right) W(\xi) .
\end{array}\right.
$$

We want to obtain the bounded solution $(U(\xi), V(\xi), W(\xi))$ of $(2.2)$ with

$$
\begin{gathered}
\lim _{\xi \rightarrow-\infty} U(\xi)=U_{0}, \lim _{\xi \rightarrow-\infty} V(\xi)=0, \lim _{\xi \rightarrow-\infty} W(\xi)=0, \\
\liminf _{\xi \rightarrow+\infty} U(\xi)>0, \liminf _{\xi \rightarrow+\infty} V(\xi)>0, \liminf _{\xi \rightarrow+\infty} W(\xi)>0,
\end{gathered}
$$

or $\liminf _{\xi \rightarrow+\infty} U(\xi)>0, \liminf _{\xi \rightarrow+\infty} V(\xi)>0(=0)$ if and only if $\liminf _{\xi \rightarrow+\infty} W(\xi)>0(=0)$. 
In particular, the solution of (2.2) with (2.3)-(2.4) is referred to as weak (persistent) (see [11]); that is, there are two positive constants $M_{1}$ and $M_{2}$ such that $M_{1}<\liminf _{\xi \rightarrow+\infty} U(\xi) \leq$ $\limsup _{\xi \rightarrow+\infty} U(\xi)<M_{2}, \quad M_{1}<\liminf _{\xi \rightarrow+\infty} V(\xi) \leq \limsup _{\xi \rightarrow+\infty} V(\xi)<M_{2}$ and $M_{1}<\liminf _{\xi \rightarrow+\infty} W(\xi) \leq$ $\limsup _{\xi \rightarrow+\infty} W(\xi)<M_{2}$.

$\xi \rightarrow+\infty$

The following assumptions are always valid throughout this article:

$\left(A_{1}\right) \quad J \in C^{1}(\mathbb{R}), J(y)=J(-y) \geq 0, J * 1=1, J$ is compactly supported and $R_{J}$ is the radius of the compact support set of $J$.

$\left(A_{2}\right) \quad G(y, s) \in C^{1}(\mathbb{R} \times[0, T])$ is Lipschitz continuous with respect to $y$ and $G(y, s)=$ $G(-y, s) \geq 0, G * 1=1$. Moreover, there exists a number $\mu_{c} \in(0,+\infty]$ such that

$$
\int_{0}^{T} \int_{-\infty}^{+\infty} G(y, s) e^{-\lambda(y+c s)} \mathrm{d} y \mathrm{~d} s<+\infty
$$

for $\lambda \in\left[0, \mu_{c}\right)$ and the wave speed $c>0$.

$\left(A_{3}\right) \quad \alpha>\max \left\{\mu+\eta_{1}+\beta, \frac{\mu+\eta_{1}+\beta}{m(c)}\right\}$ for every wave speed $c>0$, where

$$
m(c)=\inf _{0<\lambda<+\infty} \int_{0}^{T} \int_{-\infty}^{+\infty} G(y, s) e^{-\lambda(y+c s)} \mathrm{d} y \mathrm{~d} s .
$$

In fact, the above condition $\alpha>\mu+\eta_{1}+\beta$ in $\left(A_{3}\right)$ implies the basic reproduction number $\mathfrak{R}_{0}=\frac{\alpha\left(\mu+\eta_{2}+\rho+\gamma\right)}{\gamma\left(\mu+\eta_{1}\right)+\left(\mu+\eta_{1}+\beta\right)\left(\mu+\eta_{2}+\rho\right)}>1$ of the corresponding ordinary differential system of (2.1), where $\mathfrak{R}_{0}$ is well known as a threshold parameter for disease transmission models. In what follows, we just need to deal with the existence of solutions for (2.2) so as to obtain the existence of traveling waves of (2.1). As a result, the linear problem

$$
\left\{\begin{array}{l}
c V^{\prime}(\xi)=d(J * V(\xi)-V(\xi))+\alpha G * V(\xi)+\gamma W(\xi)-\left(\mu+\eta_{1}+\beta\right) V(\xi), \\
c W^{\prime}(\xi)=d(J * W(\xi)-W(\xi))+\beta V(\xi)-\gamma W(\xi)-\left(\mu+\eta_{2}+\rho\right) W(\xi)
\end{array}\right.
$$

is investigated first. Consider the solutions of the form $(V, W)=\left(q_{1}, q_{2}\right) e^{\lambda \xi}$, and the facts that $\triangle(\lambda, c)=\triangle_{1}(\lambda, c) \cdot \triangle_{2}(\lambda, c)-\gamma \beta=0, q_{1} \triangle_{1}(\lambda, c)+q_{2} \gamma=0$ and $q_{1} \beta+q_{2} \triangle_{2}(\lambda, c)=0$ can be easily examined, where

$$
\begin{aligned}
& \triangle_{1}(\lambda, c)=d \int_{-\infty}^{+\infty} J(y)\left(e^{-\lambda y}-1\right) \mathrm{d} y+\alpha \int_{0}^{T} \int_{-\infty}^{+\infty} G(y, s) e^{-\lambda(y+c s)} \mathrm{d} y \mathrm{~d} s-\left(\mu+\eta_{1}+\beta\right)-c \lambda, \\
& \triangle_{2}(\lambda, c)=d \int_{-\infty}^{+\infty} J(y)\left(e^{-\lambda y}-1\right) \mathrm{d} y-\gamma-\left(\mu+\eta_{2}+\rho\right)-c \lambda, \text { and } q_{i}>0(i=1,2) .
\end{aligned}
$$

The following results are consequently deduced:

Lemma 2.1 Supposing that $\left(A_{1}\right)-\left(A_{3}\right)$ hold, then a positive constant $c^{*}$ can be found to ensure that $\triangle(\lambda, c)=0$ admits only real roots when $c>c^{*}$. In particular, there is some positive number $\delta$ small enough satisfying $\triangle_{1}\left(\lambda_{c}+\delta, c\right)<0$ and $\triangle_{2}\left(\lambda_{c}+\delta, c\right)<0$ if $\lambda_{c}$ indicates the first positive root of $\triangle(\lambda, c)=0$. In addition, another positive constant $c_{*}$ can be found to guarantee that there exists only one positive real root of $\triangle(\lambda, c)=0$ when $0<c<c_{*}$.

Proof Let $\omega=d \int_{-\infty}^{+\infty} J(y)\left(e^{-\lambda y}-1\right) \mathrm{d} y-c \lambda$. Consider the equation $\triangle(\lambda, c)=\left[\omega+P_{1}\right][\omega+$ $\left.P_{2}\right]-\gamma \beta$, where $P_{1}=\alpha \int_{0}^{T} \int_{-\infty}^{+\infty} G(y, s) e^{-\lambda(y+c s)} \mathrm{d} y \mathrm{~d} s-\left(\mu+\eta_{1}+\beta\right)$ and $P_{2}=-\left(\gamma+\mu+\eta_{2}+\rho\right)$.

Obviously, there are two real roots of $\triangle(\lambda, c)=0$, denoted by

$$
\omega_{ \pm}=\frac{-\left(P_{1}+P_{2}\right) \pm \sqrt{\left(P_{1}-P_{2}\right)^{2}+4 \gamma \beta}}{2}
$$


Since $\alpha>\frac{\mu+\eta_{1}+\beta}{m(c)}$ for every $c>0$, this implies that $\omega_{+}>0$ and $\omega_{-}<0$. Denote

$$
N_{ \pm}(\lambda, c)=\omega-\omega_{ \pm} .
$$

Then $\triangle(\lambda, c)=N_{+}(\lambda, c) \cdot N_{-}(\lambda, c)$. Moreover, it is clear that $N_{+}(\lambda, c)=0$ admits a positive root and a negative root. Additionally, there are some $c^{*}>0$ and $\lambda_{c^{*}}>0$ satisfying that $\left.\frac{\partial N_{-}(\lambda, c)}{\partial \lambda}\right|_{\left(\lambda_{c^{*}}, c^{*}\right)}=0$ and $N_{-}\left(\lambda_{c^{*}}, c^{*}\right)=0$. At the same time, one has that

(1) there exists $\lambda_{N_{-}}^{i}>0$ such that $N_{-}\left(\lambda_{N_{-}}^{i}, c\right)=0$ for $c>c^{*}, i=1,2$;

(2) $N_{-}(\lambda, c)>0$ for $0<c<c^{*}$ and $\lambda>0$.

Therefore, there exist three positive real roots and one negative real root of $\triangle(\lambda, c)=0$ when $c>c^{*}$, and $\triangle(\lambda, c)=0$ admits only one positive real root when $0<c<c^{*}$. In addition, it is easily verified that there are some $c_{*}$ with $0<c_{*} \leq c^{*}$ and $\lambda_{c_{*}}>0$ satisfying $\triangle_{1}\left(\lambda_{c_{*}}, c_{*}\right)=0$ and $\left.\frac{\partial \triangle_{1}(\lambda, c)}{\partial \lambda}\right|_{\left(\lambda_{c_{*}}, c_{*}\right)}=0$. By a similar discussion as to that for $N_{-}(\lambda, c)$, this gives us that

(i) there exists $\lambda_{1}^{ \pm}>0$ satisfying $\triangle_{1}\left(\lambda_{1}^{ \pm}, c\right)=0$ for $c>c_{*}$;

(ii) $\triangle_{1}(\lambda, c)>0$ for $0<c<c_{*}$ and $\lambda>0$.

Accordingly, there is only one positive real root of $\triangle(\lambda, c)=0$ for $0<c<c_{*}$. Notice that the equation $\triangle_{2}(\lambda, c)=0$ admits one positive real root and one negative real root, denoted by $\lambda_{2}^{ \pm}$, for $c>c^{*}$. In addition, a simple analysis gives that

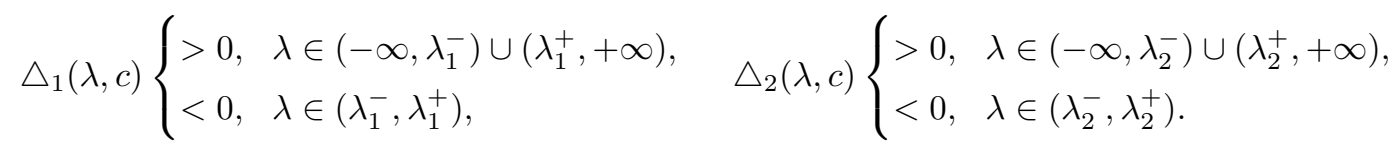

Furthermore, $\lambda_{2}^{-}<\lambda_{1}^{-}<\lambda_{1}^{+}<\lambda_{2}^{+}$and $\triangle(0, c)=P_{1} \cdot P_{2}-\gamma \beta<0$ follow. Combining the above analysis, it is obvious that

$$
\triangle_{1}(\lambda, c) \cdot \triangle_{2}(\lambda, c) \begin{cases}>0, & \lambda \in\left(-\infty, \lambda_{2}^{-}\right) \cup\left(\lambda_{1}^{-}, \lambda_{1}^{+}\right) \cup\left(\lambda_{2}^{+},+\infty\right), \\ <0, & \lambda \in\left(\lambda_{2}^{-}, \lambda_{1}^{-}\right) \cup\left(\lambda_{1}^{+}, \lambda_{2}^{+}\right) .\end{cases}
$$

Consequently, $\triangle\left(\lambda_{c}, c\right)=0$ for some $\lambda_{c}>0$ and $c>c^{*}$, where $\lambda_{c} \in\left(\lambda_{1}^{-}, \lambda_{1}^{+}\right)$and $\lambda_{c}$ is the first positive root of $\triangle(\lambda, c)=0$. Therefore, there exists some $\delta>0$ small enough such that $\triangle_{1}\left(\lambda_{c}+\delta, c\right)<0$ and $\triangle_{2}\left(\lambda_{c}+\delta, c\right)<0$, due to the fact that $\triangle_{1}(\lambda, c), \triangle_{2}(\lambda, c)$ and $\triangle(\lambda, c)$ are all continuous. The proof is complete.

Remark 2.2 For $0<c<c_{*}$, there is no real root for one of $\triangle_{1}(\lambda, c)=0$ and $\triangle_{2}(\lambda, c)=0$, at least.

\section{Existence of Traveling Waves}

In this section, we first investigate the existence and asymptotic behavior of positive bounded solutions for (2.2) with $c>c^{*}$. Second, the existence of non-negative bounded solutions for (2.2) with $c=c^{*}$ is obtained. Also, the existence and asymptotic behavior of positive bounded solutions for (2.2) with $c=c^{*}$ and $\gamma=0$ are included.

\subsection{Traveling waves for $c>c^{*}$}

In order to establish the existence and asymptotic behavior of positive bounded solutions of (2.2) for $c>c^{*}$, the following lemmas are needed: 
Lemma 3.1 The vector valued function $\left(V_{+}(x), W_{+}(x)\right):=\left(q_{1} e^{\lambda_{c} x}, q_{2} e^{\lambda_{c} x}\right)$ satisfies

$\left\{\begin{array}{l}c V_{+}^{\prime}(x)=d\left[\left(J * V_{+}\right)(x)-V_{+}(x)\right]+\alpha G * V_{+}(x)+\gamma W_{+}(x)-\left(\mu+\eta_{1}+\beta\right) V_{+}(x), \\ c W_{+}^{\prime}(x)=d\left[\left(J * W_{+}\right)(x)-W_{+}(x)\right]+\beta V_{+}(x)-\gamma W_{+}(x)-\left(\mu+\eta_{2}+\rho\right) W_{+}(x) .\end{array}\right.$

This is the direct result of system (2.6), so the proof is omitted.

Lemma 3.2 The function $U_{+}(x):=U_{0}=\frac{\Lambda}{\mu}$ satisfies the inequality

$$
c U_{+}^{\prime}(x) \geq d\left[\left(J * U_{+}\right)(x)-U_{+}(x)\right]+\Lambda-\mu U_{+}(x)-\frac{\alpha U_{+}(x) G * V_{+}(x)}{U_{+}(x)+G * V_{+}(x)+W_{+}(x)} .
$$

This is trivial, so we omit the proof.

Lemma 3.3 The function $U_{-}(x):=\max \left\{U_{0}\left(1-\kappa e^{\varsigma x}\right), 0\right\}$ satisfies the inequality

$$
c U_{-}^{\prime}(x) \leq d\left[\left(J * U_{-}\right)(x)-U_{-}(x)\right]+\Lambda-\mu U_{-}(x)-\frac{\alpha U_{-}(x) G * V_{+}(x)}{U_{-}(x)+G * V_{+}(x)}
$$

for some sufficiently small $\varsigma>0$, some large enough $\kappa>1$ and $x \neq X^{\prime}:=\frac{1}{\varsigma} \ln \frac{1}{\kappa}$.

Proof For $x<X^{\prime}$, it holds that $1-\kappa e^{\varsigma x}>0, U_{-}(x)=U_{0}\left(1-\kappa e^{\varsigma x}\right)$ and

$$
\begin{aligned}
& d\left[\left(J * U_{-}\right)(x)-U_{-}(x)\right]-c U_{-}^{\prime}(x)+\Lambda-\mu U_{-}(x)-\frac{\alpha U_{-}(x) G * V_{+}(x)}{U_{-}(x)+G * V_{+}(x)} \\
\geq & e^{\varsigma x}\left\{\mu U_{0} \kappa+U_{0} \kappa\left[c \varsigma-d \int_{-\infty}^{+\infty} J(y)\left(e^{-\varsigma y}-1\right) \mathrm{d} y\right]\right. \\
& \left.-\alpha q_{1} \kappa^{-\left(\lambda_{c}-\varsigma\right) \frac{1}{\varsigma}} \int_{0}^{T} \int_{-\infty}^{+\infty} G(y, s) e^{-\lambda_{c}(y+c s)} \mathrm{d} y \mathrm{~d} s\right\} .
\end{aligned}
$$

Keeping $\kappa \varsigma=1$ and letting $\kappa \rightarrow+\infty$, there exist $\kappa>0$ and $\varsigma>0$ such that

$$
\begin{aligned}
& \mu U_{0} \kappa+U_{0} \kappa\left[c \varsigma-d \int_{-\infty}^{+\infty} J(y)\left(e^{-\varsigma y}-1\right) \mathrm{d} y\right] \\
& -\alpha q_{1} \kappa^{-\left(\lambda_{c}-\varsigma\right) \frac{1}{\varsigma}} \int_{0}^{T} \int_{-\infty}^{+\infty} G(y, s) e^{-\lambda_{c}(y+c s)} \mathrm{d} y \mathrm{~d} s>0,
\end{aligned}
$$

so it follows that (3.2) holds.

For $x>X^{\prime}$, it is clear that $U_{-}(x)=0$ and (3.2) hold. This completes the proof.

Lemma 3.4 If $0<\varepsilon<\lambda_{c}$ is small enough and $\frac{\gamma q_{2}}{-\triangle_{1}\left(\lambda_{c}+\varepsilon, c\right)}<q_{1}<\frac{-\triangle_{2}\left(\lambda_{c}+\varepsilon, c\right) q_{2}}{\beta}$ holds, then the vector function $\left(V_{-}(x), W_{-}(x)\right)^{T}:=\left(q_{1}, q_{2}\right)^{T} e^{\lambda_{c} x} \max \left\{1-L e^{\varepsilon x}, 0\right\}$ satisfies

$$
\begin{aligned}
c V_{-}^{\prime}(x) \leq & d\left[\left(J * V_{-}\right)(x)-V_{-}(x)\right]+\frac{\alpha U_{-}(x) G * V_{-}(x)}{U_{-}(x)+G * V_{-}(x)+W_{+}(x)} \\
& +\gamma W_{-}(x)-\left(\mu+\eta_{1}+\beta\right) V_{-}(x), \\
c W_{-}^{\prime}(x) \leq & d\left[\left(J * W_{-}\right)(x)-W_{-}(x)\right]+\beta V_{-}(x)-\gamma W_{-}(x)-\left(\mu+\eta_{2}+\rho\right) W_{-}(x)
\end{aligned}
$$

for any $x \neq X^{\prime \prime}:=\frac{1}{\varepsilon} \ln \frac{1}{L}$.

Proof Taking $L>0$ large enough such that $X^{\prime \prime}<X^{\prime}$, when $x<X^{\prime \prime}\left(<X^{\prime}\right)$, we find that $V_{-}(x)=q_{1} e^{\lambda_{c} x}\left(1-L e^{\varepsilon x}\right), W_{-}(x)=q_{2} e^{\lambda_{c} x}\left(1-L e^{\varepsilon x}\right), U_{-}(x)=U_{0}\left(1-\kappa e^{\varsigma x}\right)$ and

$$
\begin{aligned}
& c V_{-}^{\prime}(x)-d\left[\left(J * V_{-}\right)(x)-V_{-}(x)\right]-\frac{\alpha U_{-}(x) G * V_{-}(x)}{U_{-}(x)+G * V_{-}(x)+W_{+}(x)} \\
& -\gamma W_{-}(x)+\left(\mu+\eta_{1}+\beta\right) V_{-}(x)
\end{aligned}
$$




$$
\begin{aligned}
= & c q_{1} \lambda_{c} e^{\lambda_{c} x}-c q_{1}\left(\lambda_{c}+\varepsilon\right) L e^{\left(\lambda_{c}+\varepsilon\right) x}-d q_{1} e^{\lambda_{c} x} \int_{-\infty}^{+\infty} J(y)\left(e^{-\lambda_{c} y}-1\right) \mathrm{d} y \\
& +d q_{1} L e^{\left(\lambda_{c}+\varepsilon\right) x} \int_{-\infty}^{+\infty} J(y)\left(e^{-\left(\lambda_{c}+\varepsilon\right) y}-1\right) \mathrm{d} y-\frac{\alpha U_{-}(x) G * V_{-}(x)}{U_{-}(x)+G * V_{-}(x)+W_{+}(x)} \\
& -\gamma q_{2} e^{\lambda_{c} x}+L q_{2} \gamma e^{\left(\lambda_{c}+\varepsilon\right) x}+\left(\mu+\eta_{1}+\beta\right) q_{1} e^{\lambda_{c} x}-q_{1} L e^{\left(\lambda_{c}+\varepsilon\right) x}\left(\mu+\eta_{1}+\beta\right) \\
= & \frac{\alpha G * V_{-}(x)\left(G * V_{-}(x)+W_{+}(x)\right)}{U_{-}(x)+G * V_{-}(x)+W_{+}(x)}+L e^{\left(\lambda_{c}+\varepsilon\right) x}\left[\triangle_{1}\left(\lambda_{c}+\varepsilon, c\right) q_{1}+\gamma q_{2}\right] .
\end{aligned}
$$

Consequently, in order to prove (3.3), it suffices to prove that

$$
\frac{\alpha G * V_{-}(x)\left(G * V_{-}(x)+W_{+}(x)\right)}{U_{-}(x)+G * V_{-}(x)+W_{+}(x)} \leq-L e^{\left(\lambda_{c}+\varepsilon\right) x}\left[\triangle_{1}\left(\lambda_{c}+\varepsilon, c\right) q_{1}+\gamma q_{2}\right],
$$

which can be guaranteed by a simple calculation for $L$ large enough. For (3.4), we have that

$$
\begin{aligned}
& c W_{-}^{\prime}(x)-d\left[\left(J * W_{-}\right)(x)-W_{-}(x)\right]-\beta V_{-}(x)+\gamma W_{-}(x)+\left(\mu+\eta_{2}+\rho\right) W_{-}(x) \\
= & -\left(q_{2} e^{\lambda_{c} x} \triangle_{2}\left(\lambda_{c}, c\right)+\beta q_{1} e^{\lambda_{c} x}\right)+q_{2} L e^{\left(\lambda_{c}+\varepsilon\right) x} \triangle_{2}\left(\lambda_{c}+\varepsilon, c\right)+\beta q_{1} L e^{\left(\lambda_{c}+\varepsilon\right) x} \\
= & L e^{\left(\lambda_{c}+\varepsilon\right) x}\left(q_{2} \triangle_{2}\left(\lambda_{c}+\varepsilon, c\right)+\beta q_{1}\right) \leq 0 .
\end{aligned}
$$

Because $V_{-}(x)=W_{-}(x)=0$ when $x>X^{\prime \prime}$, we thus have, obviously, that (3.3) and (3.4) hold. This ends the proof.

Next, we state some definitions.

Let $X>X *:=\max \left\{\left|X^{\prime}\right|,\left|X^{\prime \prime}\right|\right\}$ and define a set

$$
\Gamma_{X}=\left\{\begin{array}{l|l}
(\chi(\cdot), \varphi(\cdot), \psi(\cdot)) & \begin{array}{l}
U_{-}(\xi) \leq \chi(\xi) \leq U_{0} \\
V_{-}(\xi) \leq \varphi(\xi) \leq V_{+}(\xi)
\end{array} \quad \text { if }-X \leq \xi \leq X, \\
W_{-}(\xi) \leq \psi(\xi) \leq W_{+}(\xi) & \text { if }-X \leq \xi \leq X, \\
\chi(-X)=U_{-}(-X), & \varphi(-X)=V_{-}(-X), \\
\psi(-X)=W_{-}(-X) . &
\end{array}\right\} .
$$

For any given $(\chi(\cdot), \varphi(\cdot), \psi(\cdot)) \in \Gamma_{X}$, define

$$
\tilde{\chi}(\xi)=\left\{\begin{array}{ll}
\chi(X), & \xi>X, \\
\chi(\xi), & |\xi| \leq X, \\
U_{-}(\xi), & \xi<-X,
\end{array} \quad \tilde{\varphi}(\xi)=\left\{\begin{array}{ll}
\varphi(X), & \xi>X, \\
\varphi(\xi), & |\xi| \leq X, \\
V_{-}(\xi), & \xi<-X,
\end{array} \quad \widetilde{\psi}(\xi)= \begin{cases}\psi(X), & \xi>X, \\
\psi(\xi), & |\xi| \leq X, \\
W_{-}(\xi), & \xi<-X .\end{cases}\right.\right.
$$

In addition, a function $f: \Gamma_{X} \rightarrow C([-X, X])$ is constructed by

$$
f[\chi, \varphi, \psi](\cdot)= \begin{cases}\frac{\chi(\cdot) G * \widetilde{\varphi}(\cdot)}{\chi(\cdot)+G * \widetilde{\varphi}(\cdot)+\psi(\cdot)}, & \chi(\cdot)(G * \widetilde{\varphi}(\cdot)) \neq 0, \\ 0, & \chi(\cdot)(G * \widetilde{\varphi}(\cdot))=0,\end{cases}
$$

where $(\chi(\cdot), \varphi(\cdot), \psi(\cdot)) \in \Gamma_{X}$.

Proposition 3.5 The function $f$ is Lipschitz continuous on $\Gamma_{X}$.

Proof Choosing $\left(\chi_{1}(\cdot), \varphi_{1}(\cdot), \psi_{1}(\cdot)\right)$ and $\left(\chi_{2}(\cdot), \varphi_{2}(\cdot), \psi_{2}(\cdot)\right) \in \Gamma_{X}$ leads to

$$
\begin{aligned}
& \left|f\left[\chi_{1}, \varphi_{1}, \psi_{1}\right](\xi)-f\left[\chi_{2}, \varphi_{2}, \psi_{2}\right](\xi)\right| \\
= & \left|\frac{\chi_{1}(\xi) G * \widetilde{\varphi}_{1}(\xi)}{\chi_{1}(\xi)+G * \widetilde{\varphi}_{1}(\xi)+\psi_{1}(\xi)}-\frac{\chi_{2}(\xi) G * \widetilde{\varphi}_{2}(\xi)}{\chi_{2}(\xi)+G * \widetilde{\varphi}_{2}(\xi)+\psi_{2}(\xi)}\right|
\end{aligned}
$$




$$
\leq 2\left|G * \widetilde{\varphi}_{1}(\xi)-G * \widetilde{\varphi}_{2}(\xi)\right|+2\left|\chi_{1}(\xi)-\chi_{2}(\xi)\right|+\left|\psi_{1}(\xi)-\psi_{2}(\xi)\right| .
$$

If $\chi_{1}(\xi) G * \widetilde{\varphi}_{1}(\xi) \chi_{2}(\xi) G * \widetilde{\varphi}_{2}(\xi)=0$ and $\chi_{1}(\xi) G * \widetilde{\varphi}_{1}(\xi)+\chi_{2}(\xi) G * \widetilde{\varphi}_{2}(\xi) \neq 0$, for example, $\chi_{1}(\xi)=0$ and $\chi_{2}(\xi) G * \widetilde{\varphi}_{2}(\xi) \neq 0$, we have that

$$
\begin{aligned}
& \left|f\left[\chi_{1}, \varphi_{1}, \psi_{1}\right](\xi)-f\left[\chi_{2}, \varphi_{2}, \psi_{2}\right](\xi)\right| \\
\leq & \left|G * \widetilde{\varphi}_{1}(\xi)-G * \widetilde{\varphi}_{2}(\xi)\right|+2\left|\chi_{1}(\xi)-\chi_{2}(\xi)\right|+\left|\psi_{1}(\xi)-\psi_{2}(\xi)\right| .
\end{aligned}
$$

Other cases are similar. In addition,

$$
\begin{aligned}
\left|G * \widetilde{\varphi}_{1}(\xi)-G * \widetilde{\varphi}_{2}(\xi)\right| \leq & \mid \int_{0}^{T} \int_{-X}^{X} G(\xi-y-c s, s)\left(\varphi_{1}(y)-\varphi_{2}(y)\right) \mathrm{d} y \mathrm{~d} s \\
& +\int_{0}^{T} \int_{X}^{+\infty} G(\xi-y-c s, s)\left(\varphi_{1}(X)-\varphi_{2}(X)\right) \mathrm{d} y \mathrm{~d} s \mid \\
\leq & 2 \max _{y \in[-X, X]}\left|\varphi_{1}(y)-\varphi_{2}(y)\right| .
\end{aligned}
$$

In conclusion, the above discussions lead to

$$
\left\|f\left[\chi_{1}, \varphi_{1}, \psi_{1}\right]-f\left[\chi_{2}, \varphi_{2}, \psi_{2}\right]\right\| \leq L_{1}\left\|\varphi_{1}-\varphi_{2}\right\|+L_{2}\left\|\chi_{1}-\chi_{2}\right\|+L_{3}\left\|\psi_{1}-\psi_{2}\right\|
$$

for some $L_{1}>0, L_{2}>0, L_{3}>0$, which implies the Lipschitz continuity of $f$. This finishes the proof.

Below, for any $\xi \in[-X, X]$, consider the initial value problem

$$
\left\{\begin{array}{l}
c U^{\prime}(\xi)=d \int_{-\infty}^{+\infty} J(y) \widetilde{\chi}(\xi-y) \mathrm{d} y+\Lambda-(d+\mu) U(\xi)-\alpha f[\chi, \varphi, \psi](\xi), \\
c V^{\prime}(\xi)=d \int_{-\infty}^{+\infty} J(y) \widetilde{\varphi}(\xi-y) \mathrm{d} y-\left(d+\mu+\eta_{1}+\beta\right) V(\xi)+\gamma \psi(\xi)+\alpha f[\chi, \varphi, \psi](\xi), \\
c W^{\prime}(\xi)=d \int_{-\infty}^{+\infty} J(y) \widetilde{\psi}(\xi-y) \mathrm{d} y+\beta \varphi(\xi)-\left(d+\gamma+\mu+\eta_{2}+\rho\right) W(\xi), \\
U(-X)=U_{-}(-X), \quad V(-X)=V_{-}(-X) \quad W(-X)=W_{-}(-X) .
\end{array}\right.
$$

Naturally, (3.5) has a unique solution $U_{X}(\cdot), V_{X}(\cdot), W_{X}(\cdot) \in C^{1}([-X, X])$, by the ODE theory. Now, an operator $F=\left(F_{1}, F_{2}, F_{3}\right)$ on $\Gamma_{X}$ is defined as

$$
U_{X}(\cdot)=F_{1}(\chi(\cdot), \varphi(\cdot), \psi(\cdot)), \quad V_{X}(\cdot)=F_{2}(\chi(\cdot), \varphi(\cdot), \psi(\cdot)), \quad W_{X}(\cdot)=F_{3}(\chi(\cdot), \varphi(\cdot), \psi(\cdot)) .
$$

Theorem 3.6 The operator $F: \Gamma_{X} \rightarrow \Gamma_{X}$ is completely continuous.

Proof It is trivial to check that we have, by Lemmas 3.1-3.4, the operator $F: \Gamma_{X} \rightarrow \Gamma_{X}$. Therefore, we only need to prove the complete continuity of $F$.

Denote $U_{X, i}(\cdot)=F_{1}\left(\chi_{i}, \varphi_{i}, \psi_{i}\right), V_{X, i}(\cdot)=F_{2}\left(\chi_{i}, \varphi_{i}, \psi_{i}\right), W_{X, i}(\cdot)=F_{3}\left(\chi_{i}, \varphi_{i}, \psi_{i}\right)$ for any $\left(\chi_{i}, \varphi_{i}, \psi_{i}\right) \in \Gamma_{X}, i=1,2$. It follows from (3.5) that

$$
\begin{aligned}
U_{X}(\xi)= & U_{-}(-X) e^{-\frac{d+\mu}{c}(\xi+X)}+\frac{1}{c} \int_{-X}^{\xi} e^{\frac{d+\mu}{c}(z-\xi)}[d J * \widetilde{\chi}(z)+\Lambda-\alpha f[\chi, \varphi, \psi](z)] \mathrm{d} z, \\
V_{X}(\xi)= & V_{-}(-X) e^{-\frac{d+\mu+\eta_{1}+\beta}{c}(\xi+X)} \\
& +\frac{1}{c} \int_{-X}^{\xi} e^{\frac{d+\mu+\eta_{1}+\beta}{c}(z-\xi)}[d J * \widetilde{\varphi}(z)+\alpha f[\chi, \varphi, \psi](z)+\gamma \psi(z)] \mathrm{d} z, \\
W_{X}(\xi)= & W_{-}(-X) e^{-\frac{d+\gamma+\mu+\eta_{2}+\rho}{c}(\xi+X)}+\frac{1}{c} \int_{-X}^{\xi} e^{\frac{d+\gamma+\mu+\eta_{2}+\rho}{c}(z-\xi)}[d J * \widetilde{\psi}(z)+\beta \varphi(z)] \mathrm{d} z .
\end{aligned}
$$


Moreover

$$
\begin{aligned}
& \left|J *\left[\widetilde{\chi}_{1}(z)-\tilde{\chi}_{2}(z)\right]\right| \\
= & \left|\int_{-X}^{X} J(z-y)\left(\chi_{1}(y)-\chi_{2}(y)\right) \mathrm{d} y+\int_{X}^{+\infty} J(z-y)\left(\chi_{1}(X)-\chi_{2}(X)\right) \mathrm{d} y\right| \\
\leq & 2 \max _{y \in[-X, X]}\left|\chi_{1}(y)-\chi_{2}(y)\right| .
\end{aligned}
$$

Similarly, $\left|J * \widetilde{\varphi}_{1}(z)-J * \widetilde{\varphi}_{2}(z)\right| \leq 2 \max _{y \in[-X, X]}\left|\varphi_{1}(y)-\varphi_{2}(y)\right|$ and $\left|J * \widetilde{\psi}_{1}(z)-J * \widetilde{\psi}_{2}(z)\right| \leq$ $2 \max _{y \in[-X, X]}\left|\psi_{1}(y)-\psi_{2}(y)\right|$ follow.

In addition, the continuity of $F$ can be obtained immediately by the definitions of the operators $U_{X, i}(\cdot), V_{X, i}(\cdot), W_{X, i}(\cdot)$ and $F$. Furthermore, $U_{X}^{\prime}(\xi), V_{X}^{\prime}(\xi)$ and $W_{X}^{\prime}(\xi)$ are all bounded for $\xi \in[-X, X],(\chi(\cdot), \varphi(\cdot), \psi(\cdot)) \in \Gamma_{X}$ by $(3.5)$, and $U_{X}(\cdot), V_{X}(\cdot), W_{X}(\cdot)$ are all $C^{1}([-X, X])$, which implies that $F$ is relatively compact on $\Gamma_{X}$. Therefore, $F$ is a completely continuous operator. The proof is complete.

Obviously, $\Gamma_{X}$ is a bounded and closed convex set. It follows from Schauder's fixed-point theorem that there exists $\left(U_{X}(\xi), V_{X}(\xi), W_{X}(\xi)\right) \in \Gamma_{X}$ satisfying

$$
\left(U_{X}(\xi), V_{X}(\xi), W_{X}(\xi)\right)=F\left(\left(U_{X}(\xi), V_{X}(\xi), W_{X}(\xi)\right)\right) \text { in } \xi \in[-X, X] .
$$

Below, the existence of solutions of $(2.2)$ on $\mathbb{R}$ can be established by taking $X \rightarrow+\infty$. First, we need some priori estimates for $U_{X}(\xi), V_{X}(\xi), W_{X}(\xi)$ in the space where

$$
\begin{gathered}
C^{1,1}([-X, X]):=\left\{v \in C^{1}([-X, X]) \mid v \text { and } v^{\prime} \text { are Lipschitz continuous }\right\} \text { with the norm } \\
\|v(x)\|_{C^{1,1}([-X . X])}:=\max _{x \in[-X, X]}|v|+\max _{x \in[-X, X]}\left|v^{\prime}\right|+\sup _{x, y \in[-X, X], x \neq y} \frac{\left|v^{\prime}(x)-v^{\prime}(y)\right|}{|x-y|} .
\end{gathered}
$$

Lemma 3.7 The following two results both hold:

(i) for $X>X_{*}$, there is $M_{0}>0$ independent of $X$ satisfying $\left\|U_{X}\right\|_{C^{1}([-X, X])} \leq M_{0}$;

(ii) for each $Y>0$, there is some positive constant $M(Y)$ such that

$$
\left\|U_{X}\right\|_{C^{1,1}([-Y, Y])} \leq M(Y),\left\|V_{X}\right\|_{C^{1,1}([-Y, Y])} \leq M(Y),\left\|W_{X}\right\|_{C^{1,1}([-Y, Y])} \leq M(Y)
$$

for any $X>X_{*}$ and $X>Y+R_{J}$.

Proof (i) Obviously, $\left(U_{X}, V_{X}, W_{X}\right)$ satisfies

$$
\left\{\begin{aligned}
c U_{X}^{\prime}(\xi)= & d \int_{-\infty}^{+\infty} J(y) \widetilde{U}_{X}(\xi-y) \mathrm{d} y+\Lambda-(d+\mu) U_{X}(\xi)-\frac{\alpha U_{X}(\xi) G * \widetilde{V}_{X}(\xi)}{U_{X}(\xi)+G * \widetilde{V}_{X}(\xi)+W_{X}(\xi)} \\
c V_{X}^{\prime}(\xi)=d & \int_{-\infty}^{+\infty} J(y) \widetilde{V}_{X}(\xi-y) \mathrm{d} y-\left(d+\mu+\eta_{1}+\beta\right) V_{X}(\xi)+\gamma W_{X}(\xi) \\
& +\frac{\alpha U_{X}(\xi) G * \widetilde{V}_{X}(\xi)}{U_{X}(\xi)+G * \widetilde{V}_{X}(\xi)+W_{X}(\xi)}, \\
c W_{X}^{\prime}(\xi)= & d \int_{-\infty}^{+\infty} J(y) \widetilde{W}_{X}(\xi-y) \mathrm{d} y-\left(\gamma+d+\mu+\eta_{2}+\rho\right) W_{X}(\xi)+\beta V_{X}(\xi) .
\end{aligned}\right.
$$

Note that the first equation of (3.6) implies that $\left|U_{X}^{\prime}(\xi)\right| \leq \frac{\Lambda}{c}+\frac{2 d+\mu+\alpha}{c} U_{0}:=L_{U}$, by the facts that $U_{X} \leq U_{0}$ and $0 \leq \frac{G * \widetilde{V}_{X}(\xi)}{U_{X}(\xi)+G * \tilde{V}_{X}(\xi)+W_{X}(\xi)} \leq 1$. The proof is complete. 
(ii) For $Y \in\left(-X^{\prime \prime}, X\right)$, it follows from (i) that $\left|U_{X}(\xi)-U_{X}(\eta)\right| \leq L_{U}|\xi-\eta|$ for any $\xi, \eta \in[-X, X]$. Combining the facts that $U_{X}(x) \leq U_{0}, V_{X}(x) \leq V_{+}(Y)$ and $W_{X}(x) \leq W_{+}(Y)$ for any $x \in[-Y, Y]$, one has that

$$
\begin{aligned}
\left|V_{X}^{\prime}(\xi)\right| & \leq \frac{d}{c}\left|J * \widetilde{V}_{X}(\xi)\right|+\frac{\alpha U_{0}}{c}+\frac{\gamma}{c} W_{+}(Y)+\frac{\mu+\eta_{1}+\beta+d}{c} V_{+}(Y) \\
& \leq \frac{d}{c} V_{+}(Y)+\frac{\alpha U_{0}}{c}+\frac{\gamma}{c} W_{+}(Y)+\frac{\mu+\eta_{1}+\beta+d}{c} V_{+}(Y):=L_{V}(Y), \\
\left|W_{X}^{\prime}(\xi)\right| & \leq \frac{d}{c} W_{+}(Y)+\frac{\beta}{c} V_{+}(Y)+\frac{\gamma+d+\mu+\eta_{2}+\rho}{c} W_{+}(Y):=L_{W}(Y),
\end{aligned}
$$

which leads to $\left|V_{X}(\xi)-V_{X}(\eta)\right| \leq L_{V}(Y)|\xi-\eta|,\left|W_{X}(\xi)-W_{X}(\eta)\right| \leq L_{W}(Y)|\xi-\eta|$ for some $L_{V}(Y)>0$ and $L_{W}(Y)>0$.

By virtue of the first equation of (3.6), one has that

$$
\begin{aligned}
\left|U_{X}^{\prime}(\xi)-U_{X}^{\prime}(\eta)\right| \leq & \frac{d}{c}\left|J *\left(\widetilde{U}_{X}(\xi)-\widetilde{U}_{X}(\eta)\right)\right|+\frac{d+\mu}{c}\left|U_{X}(\xi)-U_{X}(\eta)\right| \\
& +\frac{\alpha}{c}\left|\frac{U_{X}(\xi) G * \widetilde{V}_{X}(\xi)}{U_{X}(\xi)+G * \widetilde{V}_{X}(\xi)+W_{X}(\xi)}-\frac{U_{X}(\eta) G * \widetilde{V}_{X}(\eta)}{U_{X}(\eta)+G * \widetilde{V}_{X}(\eta)+W_{X}(\eta)}\right| .
\end{aligned}
$$

By a similar discussion as to that of Theorem 2.8 in [12], and the assumption $\left(A_{1}\right)$, it follows that

$$
\begin{aligned}
& \frac{d}{c}\left|J *\left(\widetilde{U}_{X}(\xi)-\widetilde{U}_{X}(\eta)\right)\right| \\
= & \frac{d}{c} \mid \int_{-\infty}^{-X}(J(\xi-y)-J(\eta-y)) U_{-}(y) \mathrm{d} y+\int_{-X}^{X}(J(\xi-y)-J(\eta-y)) U_{X}(y) \mathrm{d} y \\
& +\int_{X}^{+\infty}(J(\xi-y)-J(\eta-y)) U_{X}(X) \mathrm{d} y \mid \\
\leq & \left.\frac{d}{c}\left(4\|J\|_{L^{\infty}} U_{0}+\kappa^{2} L_{J} U_{0} e^{-\varsigma X}+L_{U}\right)|\xi-\eta|\right),
\end{aligned}
$$

where $L_{J}$ is Lipschitz constant of $J(x)$. On the other hand, by the assumption $\left(A_{2}\right)$ and a similar discussion as to that of Lemma 8 in [22], we obtain

$$
\begin{aligned}
& \left|G * \widetilde{V}_{X}(\xi)-G * \widetilde{V}_{X}(\eta)\right| \\
\leq & T L_{G} \int_{-\infty}^{X^{\prime \prime}} V_{-}(y) \mathrm{d} y|\xi-\eta|+q_{1} e^{\lambda_{c} X} \int_{0}^{T} \int_{\eta-X-c s}^{\xi-X-c s} G(y, s) \mathrm{d} y \mathrm{~d} s \\
& +\left|\int_{0}^{T} \int_{-X}^{X}[G(\xi-y-c s, s)-G(\eta-y-c s, s)] V_{X}(y) \mathrm{d} y \mathrm{~d} s\right| \\
\leq & \left(T L_{G} \int_{-\infty}^{X^{\prime \prime}} V_{-}(y) \mathrm{d} y+3 T q_{1} e^{\lambda_{c} X}\|G\|_{L^{\infty}(\mathbb{R} \times[0, H])}+L_{V}(Y)\right)|\xi-\eta|,
\end{aligned}
$$

where $L_{G}$ is a Lipschitz constant of $G$. Moreover, it follows from Proposition 3.5 that

$$
\begin{aligned}
& \frac{\alpha}{c}\left|\frac{U_{X}(\xi) G * \widetilde{V}_{X}(\xi)}{U_{X}(\xi)+G * \widetilde{V}_{X}(\xi)+W_{X}(\xi)}-\frac{U_{X}(\eta) G * \widetilde{V}_{X}(\eta)}{U_{X}(\eta)+G * \widetilde{V}_{X}(\eta)+W_{X}(\eta)}\right| \\
\leq & \frac{\alpha}{c}\left[2 T L_{G} \int_{-\infty}^{X^{\prime \prime}} V_{-}(y) \mathrm{d} y+6 T q_{1} e^{\lambda_{c Y}}\|G\|_{L^{\infty}(\mathbb{R} \times[0, H])}+2 L_{U}+2 L_{V}(Y)+L_{W}(Y)\right]|\xi-\eta| .
\end{aligned}
$$


Combining (3.7), (3.8) and $\left|U_{X}(\xi)-U_{X}(\eta)\right| \leq L_{U}|\xi-\eta|$, we get that $\left|U_{X}^{\prime}(\xi)-U_{X}^{\prime}(\eta)\right| \leq$ $L_{U}^{\prime}(Y)|\xi-\eta|$ and

$$
\left\|U_{X}(\cdot)\right\|_{C^{1,1}([-Y, Y])} \leq U_{0}+L_{U}+L_{U}^{\prime}(Y) .
$$

Similarly, $\left\|V_{X}\right\|_{C^{1,1}([-Y, Y])} \leq M(Y)$ and $\left\|W_{X}\right\|_{C^{1,1}([-Y, Y])} \leq M(Y)$ can be obtained. This finishes the proof.

Take a sequence $\left\{X_{n}\right\}$ to be increasing and set that $\left\{X_{n}\right\}$ satisfies $X_{n}>X_{*}, X_{n}>Y+R_{J}$ and $\lim _{n \rightarrow+\infty} X_{n}=+\infty$. Then, there is some $\left(U_{X_{n}}, V_{X_{n}}, W_{X_{n}}\right) \in \Gamma_{X_{n}}$ satisfying Lemma 3.7 and (3.6) for any $n \in \mathbb{N}$. Combining Lemma 3.7 with the Arzela-Ascoli theorem, there is some convergent subsequence, denoted by $\left\{U_{X_{n_{k}}}\right\}$, converging to $\mathrm{U}$ in $C_{\text {loc }}^{1}(\mathbb{R})$. Similarly, $V_{X_{n_{k}}} \rightarrow V, W_{X_{n_{k}}} \rightarrow W$ in $C_{\text {loc }}^{1}(\mathbb{R})$, and the fact that $(U, V, W)$ satisfies $(2.2)$ can be proved.

Next, we claim that $N(\xi):=U(\xi)+V(\xi)+W(\xi) \leq \frac{\Lambda}{\mu}$ for $\xi \in \mathbb{R}$ if $(U, V, W)$ is the solution of (2.2). In reality, the equation $c v^{\prime}(\xi)=d[J * v(\xi)-v(\xi)]+\Lambda-\mu v$ has a solution $v(\xi)=\frac{\Lambda}{\mu}$. In addition, by $(2.2)$, one has that

$$
c N^{\prime}(\xi)-d[J * N-N]-(\Lambda-\mu N)=-\eta_{1} V-\left(\eta_{2}+\rho\right) W<0 .
$$

Therefore, $N(\xi) \leq \frac{\Lambda}{\mu}$ for $\xi \in \mathbb{R}$. Furthermore, the boundedness of $\|U\|_{C^{2}(\mathbb{R})},\|V\|_{C^{2}(\mathbb{R})}$ and $\|W\|_{C^{2}(\mathbb{R})}$ are obtained immediately.

Theorem 3.8 (Existence) Assume that $\left(A_{1}\right)-\left(A_{3}\right)$ hold. Then there is a bounded solution $(U(\xi), V(\xi), W(\xi))$ of $(2.2)$ satisfying $0<U(\xi)<U_{0}, V(\xi)>0, W(\xi)>0$ for every $c>c^{*}$.

Proof In light of the above discussions, we only need to show that $0<U(\xi)<U_{0}, V(\xi)>$ $0, W(\xi)>0$ for $\xi \in \mathbb{R}$.

On the contrary, if we were to suppose that $V\left(\xi_{*}\right)=0$ for some $\xi_{*}$, then

$$
d \int_{-\infty}^{+\infty} J(y) V\left(\xi_{*}-y\right) \mathrm{d} y+\frac{\alpha U\left(\xi_{*}\right) G * V\left(\xi_{*}\right)}{U\left(\xi_{*}\right)+G * V\left(\xi_{*}\right)+W\left(\xi_{*}\right)}+\gamma W\left(\xi_{*}\right)=0,
$$

which follows from the second equation of (2.2), but this is not possible, owing to the fact that $V_{-}(\xi)>0$ for $\xi<X^{\prime \prime}$, which means that $V(\xi)>0$ for all $\xi \in \mathbb{R}$. Similarly, $U(\xi)>0$ and $W(\xi)>0$ for all $\xi \in \mathbb{R}$ can be verified. Meanwhile, that $U(\xi)<U_{0}=\frac{\Lambda}{\mu}$ for any $\xi \in \mathbb{R}$ can be easily proven. In fact, if $U\left(\xi_{0}\right)=U_{0}$ for some $\xi_{0} \in \mathbb{R}$, then $U^{\prime}\left(\xi_{0}\right)=0$. Furthermore, recalling the first equation of $(2.2)$, one has that

$$
0=d\left(J * U\left(\xi_{0}\right)-U\left(\xi_{0}\right)\right)-\frac{\alpha U\left(\xi_{0}\right) G * V\left(\xi_{0}\right)}{U\left(\xi_{0}\right)+G * V\left(\xi_{0}\right)+W\left(\xi_{0}\right)}<0,
$$

which leads to a contradiction. Therefore, $U(\xi)<U_{0}$ for any $\xi \in \mathbb{R}$. The proof is ended.

Next, we demonstrate the asymptotic behavior of positive bounded solutions of (2.2) for $c>c^{*}$; that is, we prove the solutions $(U(\xi), W(\xi), V(\xi))$ of $(2.2)$ in Thoerem 3.8 satisfying (2.3) and (2.5) when $\gamma>0$, or satisfying (2.3) $-(2.4)$ when $\gamma=0$. For the proof, the following assumption $\left(A_{4}\right)$ is also needed when $\gamma=0$ :

$\left(A_{4}\right) \quad G$ is compactly supported with respect to the space variables, and $R_{G}$ is the radius of the compact support set of $G$ with $R_{J} \geq R_{G}$.

Theorem 3.9 (Asymptotic behavior) Assume that $\left(A_{1}\right)-\left(A_{3}\right)$ hold. Then, the solution $(U(\xi), V(\xi), W(\xi))$ of $(2.2)$ satisfies $\lim _{\xi \rightarrow-\infty} U(\xi)=U_{0}, \lim _{\xi \rightarrow-\infty} V(\xi)=\lim _{\xi \rightarrow-\infty} W(\xi)=0$, $\lim _{\xi \rightarrow-\infty} V(\xi) \frac{e^{-\lambda_{c} \xi}}{q_{1}}=1$ and $\lim _{\xi \rightarrow-\infty} W(\xi) \frac{e^{-\lambda_{c} \xi}}{q_{2}}=1$ for every $c>c^{*}$. Moreover, 
(i) if $\gamma>0$, then $\liminf _{\xi \rightarrow+\infty} U(\xi)>0$ and $\liminf _{\xi \rightarrow+\infty} V(\xi)>0(=0)$ if and only if $\liminf _{\xi \rightarrow+\infty} W(\xi)>$ $0(=0)$;

(ii) if $\gamma=0,\left(A_{4}\right)$ holds and $\alpha$ is large enough, then $\liminf _{\xi \rightarrow+\infty} U(\xi)>0, \liminf _{\xi \rightarrow+\infty} V(\xi)>0$ and $\liminf _{\xi \rightarrow+\infty} W(\xi)>0$.

Proof It is easy to see that $U(-\infty)=U_{0}=\frac{\Lambda}{\mu}, V(-\infty)=0$ and $W(-\infty)=0$, by the squeezing theorem. In addition, based on the fact that $V_{-}(\xi) \leq V(\xi) \leq V_{+}(\xi)$, we have that

$$
V_{-}(\xi) \frac{e^{-\lambda_{c} \xi}}{q_{1}} \leq V(\xi) \frac{e^{-\lambda_{c} \xi}}{q_{1}} \leq V_{+}(\xi) \frac{e^{-\lambda_{c} \xi}}{q_{1}} .
$$

Consequently, $\lim _{\xi \rightarrow-\infty} \frac{V(\xi) e^{-\lambda_{c} \xi}}{q_{1}}=1$, by taking $\xi \rightarrow-\infty$ in (3.9). Similarly, $\lim _{\xi \rightarrow-\infty} \frac{W(\xi) e^{-\lambda_{c} \xi}}{q_{2}}=1$ can be obtained. Next, we intend to prove (i).

Letting $U_{*}:=\liminf _{\xi \rightarrow+\infty} U(\xi), V_{*}:=\liminf _{\xi \rightarrow+\infty} V(\xi), W_{*}:=\liminf _{\xi \rightarrow+\infty} W(\xi)$, there exists some sequence $\left\{\xi_{n}\right\}_{n \in \mathbb{N}}$ with $\lim _{n \rightarrow+\infty} \xi_{n}=+\infty$ satisfying $\lim _{n \rightarrow+\infty} U\left(\xi_{n}\right)=U_{*}$. On the contrary, assume $U_{*}=0$. Let

$$
U_{n}(\xi):=U\left(\xi_{n}+\xi\right), \quad V_{n}(\xi):=V\left(\xi_{n}+\xi\right), \quad W_{n}(\xi):=W\left(\xi_{n}+\xi\right) .
$$

According to the boundedness of $U(\xi), V(\xi)$ and $W(\xi)$ in $C^{2}(\mathbb{R})$, for some functions $U_{\infty}, V_{\infty}$ and $W_{\infty}$, we can extract some subsequences of $\left\{U_{n}(\xi)\right\},\left\{V_{n}(\xi)\right\}$ and $\left\{W_{n}(\xi)\right\}$, respectively, still denoted by $\left\{U_{n}(\xi)\right\},\left\{V_{n}(\xi)\right\}$ and $\left\{W_{n}(\xi)\right\}$, satisfying $U_{n}(\xi) \rightarrow U_{\infty}(\xi), V_{n}(\xi) \rightarrow V_{\infty}(\xi)$ and $W_{n}(\xi) \rightarrow W_{\infty}(\xi)$ in $C_{\text {loc }}^{1}(\mathbb{R})$ as $n \rightarrow+\infty$, where $U_{\infty}(0)=0, U_{\infty}^{\prime}(0)=0$. This implies that $d \int_{\mathbb{R}} J(y) U_{\infty}(-y) \mathrm{d} y+\Lambda=0$, from the first equation of (2.2), which is impossible, since $\Lambda>0$. Hence, $U_{*}>0$.

It is not difficult to derive that $V_{*}>0$ if and only if $W_{*}>0$. First, we prove that $V_{*}=0$ if $W_{*}=0$. For the case of $W_{*}=0$, there is some sequence $\left\{\eta_{n}\right\}_{n \in \mathbb{N}}$ with $\lim _{n \rightarrow+\infty} \eta_{n}=+\infty$ satisfying $\lim _{n \rightarrow+\infty} W\left(\eta_{n}\right)=W_{*}=0$ and $\lim _{n \rightarrow+\infty} W^{\prime}\left(\eta_{n}\right)=0$. Similarly, taking $U_{n}(\xi):=U\left(\eta_{n}+\right.$ $\xi), \quad V_{n}(\xi):=V\left(\eta_{n}+\xi\right)$ and $W_{n}(\xi):=W\left(\eta_{n}+\xi\right)$, we can claim that there are some functions $\widetilde{U}_{\infty}(\xi), \widetilde{V}_{\infty}(\xi), \widetilde{W}_{\infty}(\xi)$ satisfying that

$$
U_{n}(\xi) \rightarrow \widetilde{U}_{\infty}(\xi), \quad V_{n}(\xi) \rightarrow \widetilde{V}_{\infty}(\xi), \quad W_{n}(\xi) \rightarrow \widetilde{W}_{\infty}(\xi) \quad \text { in } \quad C_{\mathrm{loc}}^{1}(\mathbb{R})
$$

as $n \rightarrow+\infty$. Hence, $\widetilde{W}_{\infty}(0)=\widetilde{W}_{\infty}^{\prime}(0)=0$. In addition, $d \int_{\mathbb{R}} J(y) \widetilde{W}_{\infty}(-y) \mathrm{d} y+\beta \widetilde{V}_{\infty}(0)=0$, by the third equation of $(2.2)$, which leads to $\widetilde{V}_{\infty}(0)=0$. Owing to the fact that $\widetilde{V}_{\infty}(0)=$ $\lim _{n \rightarrow+\infty} V\left(\eta_{n}\right) \geq V_{*}$, one can get $V_{*}=0$. Similarly, we obtain $W_{*}=0$ if $V_{*}=0$. Hence, $V_{*}=0$ if and only if $W_{*}=0$; that is, $V_{*}>0$ if and only if $W_{*}>0$. Therefore, (i) is proved.

Below, we intend to show that (ii) is valid. It only needs to be verified that $V_{*}>0$. To this end, we claim that $V^{\prime}(\xi)>0$ for any $\xi \in \mathbb{R}$ if $V(\xi) \leq \varepsilon_{0}$ for some constant $\varepsilon_{0}>0$ small enough. Note that $V_{*}>0$ if the claim is true. By contrast, suppose that there is a sequence $\left\{\widetilde{\xi}_{n}\right\}_{n \in \mathbb{N}}$ satisfying $\lim _{n \rightarrow+\infty} V\left(\widetilde{\xi}_{n}\right)=0$ and $V^{\prime}\left(\widetilde{\xi}_{n}\right) \leq 0$ for all $n \in \mathbb{N}$. Let $U_{n}(\xi):=$ $U\left(\widetilde{\xi}_{n}+\xi\right), \quad V_{n}(\xi):=V\left(\widetilde{\xi}_{n}+\xi\right), \quad W_{n}(\xi):=W\left(\widetilde{\xi}_{n}+\xi\right)$. Therefore, $\lim _{n \rightarrow+\infty} V_{n}(0)=0$ and $\lim _{n \rightarrow+\infty} V_{n}(\xi)=0$ in $C_{\text {loc }}^{1}(\mathbb{R})$. Recalling the second equation of $(2.2)$, one has that $c\left|V_{n}^{\prime}(\xi)\right| \leq$ $d \int_{-\infty}^{+\infty} J(y) V_{n}(\xi-y) \mathrm{d} y+\left(d+\mu+\eta_{1}+\beta\right) V_{n}(\xi)+\alpha G * V_{n}(\xi)$, which implies that $\lim _{n \rightarrow+\infty} V_{n}^{\prime}(\xi)=0$ in $C_{\text {loc }}^{1}(\mathbb{R})$. 
By the first equation of $(2.2)$, it follows that

$$
c U_{n}^{\prime}(\xi)=d\left(J * U_{n}(\xi)-U_{n}(\xi)\right)+\Lambda-\mu U_{n}(\xi)-\frac{\alpha U_{n}(\xi) G * V_{n}(\xi)}{U_{n}(\xi)+G * V_{n}(\xi)+W_{n}(\xi)} .
$$

In view of the boundedness of $\left\|U_{n}\right\|_{C^{2}}$, there is some function $U_{\infty}(\xi)$ such that $U_{n}(\xi) \rightarrow U_{\infty}(\xi)$ uniformly in $C_{\text {loc }}^{1}(\mathbb{R})$, by extracting a subsequence of $\left\{U_{n}(\xi)\right\}$, still denoted by $\left\{U_{n}(\xi)\right\}$, and such that $U_{\infty}(\xi)$ satisfies

$$
c U_{\infty}^{\prime}(\xi)=d\left(J * U_{\infty}(\xi)-U_{\infty}(\xi)\right)+\Lambda-\mu U_{\infty}(\xi) .
$$

Setting $\tau_{0}=\inf _{\xi \in \mathbb{R}} U_{\infty}(\xi), \lim _{m \rightarrow+\infty} U_{\infty}\left(\zeta_{m}\right)=\tau_{0}$ for some sequence $\left\{\zeta_{m}\right\}_{m \in \mathbb{N}}$. Without loss of generality, there exists some function $\varphi_{\infty}(\xi)$ such that $\varphi_{m}(\xi):=U_{\infty}\left(\xi+\zeta_{m}\right) \rightarrow \varphi_{\infty}(\xi)$ uniformly in $C_{\text {loc }}^{1}(\mathbb{R})$, by extracting a subsequence of $\left\{\varphi_{m}(\xi)\right\}$, still denoted by $\left\{\varphi_{m}(\xi)\right\}$, where $\varphi_{\infty}(\xi)$ satisfies (3.10) and $\varphi_{\infty}(0)=\tau_{0}, \varphi_{\infty}^{\prime}(0)=0$. In addition, it is easy to see from (3.10) that $\Lambda-\mu \varphi_{\infty}(0) \leq 0$, which implies that $\varphi_{\infty}(0) \geq \frac{\Lambda}{\mu}$. Moreover, we obtain $\varphi_{\infty}(0)=\frac{\Lambda}{\mu}$, due to the fact that $U_{\infty} \leq \frac{\Lambda}{\mu}$. Therefore, $U_{\infty}(\xi)=\frac{\Lambda}{\mu}$ on $\mathbb{R}$.

Applying a similar discussion to Lemma 2.2 in [10], there exists a positive number $C$ independent of $n$ such that $\frac{V_{n}^{\prime}(\xi)}{V_{n}(\xi)}<C$ and $\int_{\mathbb{R}} J(y) \frac{V_{n}(\xi-y)}{V_{n}(\xi)} \mathrm{d} y<C$. Denoting $\psi_{n}(\xi):=\frac{V_{n}(\xi)}{V_{n}(0)}=$ $e^{\int_{0}^{\xi} \frac{V_{n}^{\prime}(z)}{V_{n}(z)} \mathrm{d} z}$, it is not difficult to obtain that $\psi_{n}(\xi)$ is locally uniform bounded on $\mathbb{R}$, and thus the local uniform boundedness of $\psi_{n}^{\prime}(\xi)$ can follow due to the fact that $\psi_{n}^{\prime}(\xi)=\frac{V_{n}^{\prime}(\xi)}{V_{n}(\xi)} \psi_{n}(\xi)$. Letting $n \rightarrow+\infty$, there exists some function $\psi_{\infty}(\xi)$ such that

$$
\begin{aligned}
c \psi_{\infty}^{\prime}(\xi)= & d\left[J * \psi_{\infty}(\xi)-\psi_{\infty}(\xi)\right]+\frac{\alpha U_{\infty}(\xi)}{U_{\infty}(\xi)+G * V_{\infty}(\xi)+W_{\infty}(\xi)} G * \psi_{\infty}(\xi) \\
& -\left(\mu+\eta_{1}+\beta\right) \psi_{\infty}(\xi) .
\end{aligned}
$$

We claim that $\psi_{\infty}(\xi)>0$ on $\mathbb{R}$. If we were to assume, by contrast, that $\psi_{\infty}\left(\xi_{0}\right)=0$ for some $\xi_{0} \in \mathbb{R}$, then $\psi_{\infty}^{\prime}\left(\xi_{0}\right)=0$. According to (3.11), it holds that

$$
0=d \int_{\mathbb{R}} J(y) \psi_{\infty}\left(\xi_{0}-y\right) \mathrm{d} y+\frac{\alpha U_{\infty}\left(\xi_{0}\right) G * \psi_{\infty}\left(\xi_{0}\right)}{U_{\infty}\left(\xi_{0}\right)+G * V_{\infty}\left(\xi_{0}\right)+W_{\infty}\left(\xi_{0}\right)},
$$

which implies that $\psi_{\infty}(\xi) \equiv 0$. This leads to a contradiction with $\psi_{\infty}(0)=1$. Denote $Z(\xi):=$ $\frac{\psi_{\infty}^{\prime}(\xi)}{\psi_{\infty}(\xi)}$, then $Z(\xi)$ satisfies that

$$
\begin{aligned}
c Z(\xi)= & d \int_{\mathbb{R}} J(y) e^{\int_{\xi}^{\xi-y} Z(\eta) \mathrm{d} \eta} \mathrm{d} y-d+\frac{\alpha U_{\infty}(\xi)}{U_{\infty}(\xi)+G * V_{\infty}(\xi)+W_{\infty}(\xi)} \\
& \iint_{D} G(y, s) e^{\int_{\xi}^{\xi-(y+c s)} Z(\eta) \mathrm{d} \eta} \mathrm{d} y \mathrm{~d} s-\left(\mu+\eta_{1}+\beta\right),
\end{aligned}
$$

where $D=\left\{(y, s) \mid-R_{G} \leq y \leq R_{G}, 0<s \leq T\right\}$. It is not difficult to examine $Z(\xi)>$ $-\frac{d+\mu+\eta_{1}+\beta}{c}:=-C_{1}$. Furthermore, defining $D_{1}=\left\{(y, s) \in D \mid-R_{G} \leq y+c s \leq R_{G}\right\}$ and $D_{2}=\left\{(y, s) \in D \mid-R_{G} \leq y+c s \leq 0\right\}$, we get that $D_{2} \subset D_{1} \subset D$ and

$$
\iint_{D} G(y, s) e^{\int_{\xi}^{\xi-(y+c s)} Z(\eta) \mathrm{d} \eta} \mathrm{d} y \mathrm{~d} s \geq \iint_{D_{2}} G(y, s) e^{\int_{\xi}^{\xi-(y+c s)} Z(\eta) \mathrm{d} \eta} \mathrm{d} y \mathrm{~d} s>0,
$$

and thereby $Z(\xi)>0$ can be guaranteed by choosing

$$
\alpha>b(c):=\frac{2\left(d+\mu+\eta_{1}+\beta-d \int_{-\infty}^{0} J(y) e^{C_{1} y} \mathrm{~d} y\right)}{\iint_{D_{2}} G(y, s) e^{(y+c s) C_{1}} \mathrm{~d} y \mathrm{~d} s} .
$$


Combining the fact that $\psi_{\infty}(\xi)>0$ with the definition of $Z(\xi)$, one has $\psi_{\infty}^{\prime}(\xi)>0$ and

$$
0<\psi_{\infty}^{\prime}(0)=\lim _{n \rightarrow+\infty} \psi_{n}^{\prime}(0)=\lim _{n \rightarrow+\infty} \frac{V_{n}^{\prime}(0)}{V_{n}(0)} .
$$

Therefore, $V^{\prime}\left(\widetilde{\xi_{n}}\right)=V_{n}^{\prime}(0)>0$, which is a contradiction. This completes the proof.

\subsection{Traveling waves for $c=c^{*}$}

In this subsection, the existence and asymptotic behavior of solutions of (2.2) for wave speed $c=c^{*}$ are investigated. It is easy to verify that (2.1) admits a unique positive equilibrium $\mathbf{E}^{*}:=\left(U^{*}, V^{*}, W^{*}\right)$ when $\gamma=0$ and $\left(A_{3}\right)$ holds, where $U^{*}>0, V^{*}>0, W^{*}>0$. For the proof, the following result is necessary:

Lemma 3.10 Assume that $\left(A_{1}\right)-\left(A_{3}\right)$ hold. Then, for every $c>c^{*}$, both $U(+\infty)$ and $W(+\infty)$ exist, and $(U(\xi), V(\xi), W(\xi))$ satisfies $(U(+\infty), V(+\infty), W(+\infty))=\mathbf{E}^{*}$ if $\gamma=0$, $\alpha>b(c)$ and $V(+\infty)$ exists.

Proof Suppose that $\gamma=0$ and $\lim _{\xi \rightarrow+\infty} V(\xi)=V_{0}$. It follows from Theorem 3.9 that $V_{0}>0$. We claim that $W(+\infty)$ exists. Otherwise, $\underline{W}:=\liminf _{\xi \rightarrow+\infty} W(\xi)<\limsup _{\xi \rightarrow+\infty} W(\xi):=\bar{W}$. According to the Fluctuation Lemma, there exist some sequences $\left\{\xi_{n}^{1}\right\}$ and $\left\{\xi_{n}^{2}\right\}$ satisfying $\lim _{n \rightarrow+\infty} \xi_{n}^{1} \rightarrow+\infty$ and $\lim _{n \rightarrow+\infty} \xi_{n}^{2} \rightarrow+\infty$ such that

$$
\lim _{n \rightarrow+\infty} W\left(\xi_{n}^{1}\right)=\underline{W}, W^{\prime}\left(\xi_{n}^{1}\right)=0 \text { and } \lim _{n \rightarrow+\infty} W\left(\xi_{n}^{2}\right)=\bar{W}, W^{\prime}\left(\xi_{n}^{2}\right)=0 .
$$

By the third equation of (2.2), we obtain that

$$
\left\{\begin{array}{l}
0=c W^{\prime}\left(\xi_{n}^{1}\right)=d\left(J * W\left(\xi_{n}^{1}\right)-W\left(\xi_{n}^{1}\right)\right)+\beta V\left(\xi_{n}^{1}\right)-\left(\mu+\eta_{2}+\rho\right) W\left(\xi_{n}^{1}\right), \\
0=c W^{\prime}\left(\xi_{n}^{2}\right)=d\left(J * W\left(\xi_{n}^{2}\right)-W\left(\xi_{n}^{2}\right)\right)+\beta V\left(\xi_{n}^{2}\right)-\left(\mu+\eta_{2}+\rho\right) W\left(\xi_{n}^{2}\right),
\end{array}\right.
$$

and it follows that $\beta V_{0}-\left(\mu+\eta_{2}+\rho\right) \bar{W} \geq 0 \geq \beta V_{0}-\left(\mu+\eta_{2}+\rho\right) \underline{W}$, that is, $\bar{W} \leq \underline{W}$, which leads to a contradiction. Combining this with the fact that $\liminf _{\xi \rightarrow+\infty} W(\xi)>0$ leads to $W(+\infty):=W_{0}>0$.

On the other hand, for any sequence $\left\{\xi_{n}\right\}_{n=1}^{+\infty}$ satisfying $\xi_{n} \rightarrow+\infty$ as $n \rightarrow+\infty$, define

$$
U_{n}(\xi):=U\left(\xi_{n}+\xi\right), \quad V_{n}(\xi):=V\left(\xi_{n}+\xi\right) \text { and } W_{n}(\xi):=W\left(\xi_{n}+\xi\right) .
$$

Then, there exists some function $U_{\infty}(\xi)$, up to extracting some subsequences as necessary, such that $U_{n}(\xi) \rightarrow U_{\infty}(\xi), V_{n}(\xi) \rightarrow V_{0}, W_{n}(\xi) \rightarrow W_{0}$ in $C_{\text {loc }}^{1}(\mathbb{R})$ as $n \rightarrow+\infty$, by the boundedness of $\left\|U_{n}\right\|_{C^{2}}$. By the second equation of (2.2), one has that

$$
c V_{n}^{\prime}(\xi)=d\left(J * V_{n}(\xi)-V_{n}(\xi)\right)+\frac{\alpha U_{n}(\xi) G * V_{n}(\xi)}{U_{n}(\xi)+G * V_{n}(\xi)+W_{n}(\xi)}-\left(\mu+\eta_{1}+\beta\right) V_{n}(\xi) .
$$

Furthermore, $0=\frac{\alpha U_{\infty}(\xi) V_{0}}{U_{\infty}(\xi)+V_{0}+W_{0}}-\left(\mu+\eta_{1}+\beta\right) V_{0}$, by letting $n \rightarrow+\infty$, which implies that there exists some positive number $U_{1}>0$ such that $U_{\infty}(\xi) \equiv U_{1}$ on $\mathbb{R}$, so $\lim _{\xi \rightarrow+\infty} U(\xi)=U_{1}$, by the arbitrariness of $\left\{\xi_{n}\right\}$. In addition, $\left(U_{1}, V_{0}, W_{0}\right)$ is a equilibrium of $(2.1)$, due to the fact that $\left(U_{\infty}(\xi), V_{0}, W_{0}\right)$ satisfies $(2.2)$. Therefore, $\left(U_{1}, V_{0}, W_{0}\right)=E^{*}$, owing to the facts that $U_{1}>0, V_{0}>0, W_{0}>0$ and to the uniqueness of positive equilibrium. The proof is finished.

Theorem 3.11 Assume that $\left(A_{1}\right)-\left(A_{3}\right)$ hold. Then, for $c=c^{*}$ and $\gamma \geq 0$, there is a bounded solution $(U(\xi), V(\xi), W(\xi))$ of $(2.2)$ satisfying $0 \leq U(\xi) \leq U_{0}, V(\xi) \geq 0$ and $W(\xi) \geq 0$ 
for all $\xi \in \mathbb{R}$. In particular, if $\gamma=0$, then the solution of (2.2) satisfies $0<U(\xi)<U_{0}, V(\xi)>0$ and $W(\xi)>0$ for all $\xi \in \mathbb{R}$. In addition, the solution of (2.2) satisfies $(2.3)-(2.4)$ if $\gamma=$ $0, \alpha>b(c)$ and $\left(A_{4}\right)$ holds.

Proof Take some sequence $\left\{c_{n}\right\}$ such that $c^{*}<c_{n} \leq c^{*}+1$ with $c_{n}>c_{n+1}$ for all $n \in \mathbb{N}$ and $\lim _{n \rightarrow+\infty} c_{n}=c^{*}$. Suppose that $\left(U_{n}, V_{n}, W_{n}\right)$ is the bounded solution of (2.2), associated with $c_{n}$. Then $0<U_{n}(\xi)<U_{0}, V_{n}(\xi)>0$ and $W_{n}(\xi)>0$ follow from Theorem 3.8. We can see that there exists some subsequence $\left(U_{n}(\xi), V_{n}(\xi), W_{n}(\xi)\right)$ converging to $(U(\xi), V(\xi), W(\xi))$ in $C_{\text {loc }}^{1}$ by the uniform boundedness of $U_{n}(\xi), V_{n}(\xi)$ and $W_{n}(\xi)$ in $C^{2}(\mathbb{R})$. Furthermore, $(U(\xi), V(\xi), W(\xi))$ satisfies (2.2) with $c=c^{*}$ and

$$
0 \leq U(\xi) \leq U_{0}, V(\xi) \geq 0, W(\xi) \geq 0, \text { for all } \xi \in \mathbb{R}
$$

For $\gamma=0$, it is necessary to re-establish the existence of a positive bounded solution for (2.2). Note that when $\gamma=0, V^{\prime}(\xi)>0$ for any $\xi \in \mathbb{R}$ if $V(\xi) \leq \varepsilon_{0}$ for some $\varepsilon_{0}>0$. Therefore, we know that $\liminf _{n \rightarrow+\infty}\left\|V_{n}\right\|_{L^{\infty}(\mathbb{R})}>0$, by a similar discussion as to that regarding Theorem 3.19 in [11]. Moreover, it follows from the fact $V_{n}(-\infty)=0$ that there exists some $\xi_{n} \in \mathbb{R}$ so that $V_{n}\left(\xi_{n}\right)=\tau^{*}$ for some sufficiently small $\tau^{*}$ with $0<\tau^{*}<\min \left\{\varepsilon_{0}, V^{*}\right\}$.

Denote $\widetilde{U}_{n}(\xi):=U_{n}\left(\xi+\xi_{n}\right), \quad \widetilde{V}_{n}(\xi):=V_{n}\left(\xi+\xi_{n}\right)$ and $\widetilde{W}_{n}(\xi):=W_{n}\left(\xi+\xi_{n}\right)$ for all $\xi \in \mathbb{R}$. Assume that $\widetilde{V}_{n}(\xi) \leq \tau^{*}$ for all $\xi<0$. Then, there are some functions $U(\xi), V(\xi)$ and $W(\xi)$, satisfying $\lim _{n \rightarrow+\infty}\left(\widetilde{U}_{n}(\xi), \widetilde{V}_{n}(\xi), \widetilde{W}_{n}(\xi)\right)=(U(\xi), V(\xi), W(\xi))$ in $C_{\text {loc }}^{1}(\mathbb{R})$, that are achieved by extracting some subsequences of $\widetilde{U}_{n}(\xi), \widetilde{V}_{n}(\xi)$ and $\widetilde{W}_{n}(\xi)$, respectively, still denoted by $\widetilde{U}_{n}(\xi), \widetilde{V}_{n}(\xi)$ and $\widetilde{W}_{n}(\xi)$. Here, $(U, V, W)$ is a solution of $(2.2)$ for $c=c^{*}$ and $V(0)=\tau^{*}$. Below, we are going to check that $0<U(\xi)<U_{0}, V(\xi)>0$ and $W(\xi)>0$ for all $\xi \in \mathbb{R}$.

Arguing by contradiction, if $V\left(\xi_{0}^{\prime}\right)=0$ for some $\xi_{0}^{\prime} \in \mathbb{R}$, then $V^{\prime}\left(\xi_{0}^{\prime}\right)=0$. The second equation of (2.2) implies that $0=d \int_{\mathbb{R}} J(y) V\left(\xi_{0}^{\prime}-y\right) \mathrm{d} y+\frac{\alpha U\left(\xi_{0}^{\prime}\right) G * V\left(\xi_{0}^{\prime}\right)}{U\left(\xi_{0}^{\prime}\right)+G * V\left(\xi_{0}^{\prime}\right)+W\left(\xi_{0}^{\prime}\right)}$. This leads to $V(\xi) \equiv 0$ on $\mathbb{R}$, which contradicts with $V(0)=\tau^{*}>0$. Therefore, $V(\xi)>0$. Moreover, $0<U(\xi)<U_{0}$ and $W(\xi)>0$ can be obtained by a method similar to that of Theorem 3.8.

Finally, we intend to show that the solution of (2.2) satisfies (2.3)-(2.4) when $c=c^{*}$ and $\gamma=0$. A similar discussion as to that concerning Theorem 3.9 gives that

$$
\liminf _{\xi \rightarrow+\infty} U(\xi)>0, \liminf _{\xi \rightarrow+\infty} V(\xi)>0, \liminf _{\xi \rightarrow+\infty} W(\xi)>0, \text { and } V^{\prime}(\xi)>0 \text { for } \xi<0 .
$$

Therefore, $V(-\infty):=\lim _{\xi \rightarrow-\infty} V(\xi)$ exists, by the boundedness of $V(\xi)$. We claim that $V(-\infty)=$ 0 . Assume by contrast that $V(-\infty)>0$. Then $V(-\infty)=V^{*}>\tau^{*}$, which follows from Lemma 3.10, which is in contradiction with the fact that $V(-\infty) \leq \tau^{*}$. Hence, $V(-\infty)=0$. Furthermore, $\lim _{\xi \rightarrow-\infty} U(\xi)=U_{0}, \lim _{\xi \rightarrow-\infty} W(\xi)=0$ can be obtained by a similar method as to that in Lemma 3.10. The proof is complete.

Remark 3.12 The existence and asymptotic behavior of positive bounded traveling waves with $c=c^{*}$ for $\gamma=0$ can be included in Theorem 3.11. However, the existence and asymptotic behavior of positive bounded traveling waves with $c=c^{*}$ for $\gamma>0$ are still open questions.

\section{Nonexistence of Traveling Waves}

In this section, we intend to establish the nonexistence of solution of (2.2). 
Theorem 4.1 Assume that $\left(A_{1}\right)-\left(A_{3}\right)$ hold. Then there are no bounded nontrivial positive solutions of (2.2) satisfying $(2.3)-(2.4)$ for every $0<c<c_{*}$.

Proof Taking the contrary, suppose that there exists a bounded nontrivial positive solution $(U(\xi), V(\xi), W(\xi))$ of $(2.2)$ with $(2.3)-(2.4)$ for some $0<c<c_{*}$, so $U(-\infty)=U_{0}$, $V(-\infty)=0, W(-\infty)=0$. Then, $\lim _{\xi \rightarrow-\infty} \frac{\alpha U(\xi)}{U(\xi)+G * V(\xi)+W(\xi)}=\alpha$, and there is a number $\xi^{*}$ such that $\frac{\alpha U(\xi)}{U(\xi)+G * V(\xi)+W(\xi)}>\frac{\alpha+\mu+\eta_{1}+\beta}{2}$ for any $\xi<\xi^{*}$. In addition, the second equation of $(2.2)$ implies that

$$
c V^{\prime}(\xi) \geq d[(J * V)(\xi)-V(\xi)]+\frac{\alpha+\mu+\eta_{1}+\beta}{2}[G * V(\xi)-V(\xi)]+\frac{\alpha-\mu-\eta_{1}-\beta}{2} V(\xi) .
$$

Integrating (4.1) from $-\infty$ to $\xi$, where $\xi<\xi^{*}$, one has that

$$
\begin{aligned}
c[V(\xi)-V(-\infty)] \geq & d \int_{-\infty}^{\xi}[(J * V)(\eta)-V(\eta)] \mathrm{d} \eta+\frac{\alpha-\mu-\eta_{1}-\beta}{2} \int_{-\infty}^{\xi} V(\eta) \mathrm{d} \eta \\
& +\frac{\alpha+\mu+\eta_{1}+\beta}{2} \int_{-\infty}^{\xi}[G * V(\eta)-V(\eta)] \mathrm{d} \eta .
\end{aligned}
$$

Define $Q(\xi):=\int_{-\infty}^{\xi} V(\eta) \mathrm{d} \eta$. It is obvious that $\int_{-\infty}^{\xi}(J * V)(\eta) \mathrm{d} \eta=J * Q(\xi)$ and $\int_{-\infty}^{\xi}(G * V)(\eta) \mathrm{d} \eta=G * Q(\xi)$, so it follows from (4.2) that

$$
\frac{\alpha-\mu-\eta_{1}-\beta}{2} Q(\xi) \leq c V(\xi)-d[J * Q(\xi)-Q(\xi)]-\frac{\alpha+\mu+\eta_{1}+\beta}{2}[G * Q(\xi)-Q(\xi)] .
$$

Integrating (4.3) from $-\infty$ to $\xi$, where $\xi<\xi^{*}$, this leads to

$$
\begin{aligned}
\frac{\alpha-\mu-\eta_{1}-\beta}{2} \int_{-\infty}^{\xi} Q(\eta) \mathrm{d} \eta \leq & c \int_{-\infty}^{\xi} V(\eta) \mathrm{d} \eta-d \int_{-\infty}^{\xi}[J * Q(\eta)-Q(\eta)] \mathrm{d} \eta \\
& -\frac{\alpha+\mu+\eta_{1}+\beta}{2} \int_{-\infty}^{\xi}[G * Q(\eta)-Q(\eta)] \mathrm{d} \eta .
\end{aligned}
$$

By a similar calculation as to that of Wang [19], we obtain

$$
\begin{aligned}
& \int_{-\infty}^{\xi}[J * Q(\eta)-Q(\eta)] \mathrm{d} \eta=-\int_{-\infty}^{+\infty} y J(y) \int_{0}^{1} Q(\xi-\theta y) \mathrm{d} \theta \mathrm{d} y \\
& \int_{-\infty}^{\xi}[G * Q(\eta)-Q(\eta)] \mathrm{d} \eta=-\int_{0}^{T} \int_{-\infty}^{+\infty}(y+c s) G(y, s) \int_{0}^{1} Q(\xi-\theta(y+c s)) \mathrm{d} \theta \mathrm{d} y \mathrm{~d} s .
\end{aligned}
$$

Substituting (4.5) and (4.6) into (4.4), one has that

$$
\begin{aligned}
& \frac{\alpha-\mu-\eta_{1}-\beta}{2} \int_{-\infty}^{\xi} Q(\eta) \mathrm{d} \eta \\
\leq & c Q(\xi)+d \int_{-\infty}^{+\infty} y J(y) \int_{0}^{1} Q(\xi-\theta y) \mathrm{d} \theta \mathrm{d} y \\
& +\frac{\alpha+\mu+\eta_{1}+\beta}{2} \int_{0}^{T} \int_{-\infty}^{+\infty}(y+c s) G(y, s) \int_{0}^{1} Q(\xi-\theta(y+c s)) \mathrm{d} \theta \mathrm{d} y \mathrm{~d} s .
\end{aligned}
$$

Notice that $y Q(\xi-\theta y)$ is decreasing with respect to $\theta \in[0,1]$ for every $y \in \mathbb{R}$, that is, $y Q(\xi-\theta y) \leq y Q(\xi)$. Similarly, the fact that $(y+c s) Q(\xi-\theta(y+c s)) \leq(y+c s) Q(\xi)$ can be obtained. In addition, $\int_{-\infty}^{+\infty} J(y) y \mathrm{~d} y=0$ and $\int_{0}^{T} \int_{-\infty}^{+\infty} G(y, s) y \mathrm{~d} y \mathrm{~d} s=0$ can be derived from $\left(A_{1}\right)-\left(A_{2}\right)$. Hence, based on (4.7), we get that

$$
\frac{\alpha-\mu-\eta_{1}-\beta}{2} \int_{-\infty}^{\xi} Q(\eta) \mathrm{d} \eta
$$




$$
\begin{aligned}
& \leq c Q(\xi)+d \int_{-\infty}^{+\infty} y J(y) Q(\xi) \mathrm{d} y+\frac{\alpha+\mu+\eta_{1}+\beta}{2} \int_{0}^{T} \int_{-\infty}^{+\infty}(y+c s) G(y, s) Q(\xi) \mathrm{d} y \mathrm{~d} s \\
& <c^{*}\left[1+\frac{\alpha+\mu+\eta_{1}+\beta}{2} \int_{0}^{T} \int_{-\infty}^{+\infty} s G(y, s) \mathrm{d} y \mathrm{~d} s\right] \cdot Q(\xi):=C_{2} Q(\xi) .
\end{aligned}
$$

Since $Q(\xi)$ is increasing due to $Q^{\prime}(\xi)=V(\xi)>0$, one has that

$$
\int_{-\infty}^{\xi} Q(t) \mathrm{d} t=\int_{0}^{+\infty} Q(\xi-t) \mathrm{d} t \geq \int_{0}^{\omega_{0}} Q(\xi-t) \mathrm{d} t>\omega_{0} Q\left(\xi-\omega_{0}\right)
$$

for any $\omega_{0}>0$. Therefore, it follows from (4.8)-(4.9) that $\frac{\alpha-\mu-\eta_{1}-\beta}{2} \omega_{0} Q\left(\xi-\omega_{0}\right)<C_{2} Q(\xi)$ for $\xi<$ $\xi^{*}$, therefore, there is some $\omega_{1}$ satisfying that $Q\left(\xi-\omega_{1}\right)<\frac{Q(\xi)}{2}$. Denote $K(\xi)=Q(\xi) e^{-\bar{\mu}_{0} \xi}$ for $\bar{\mu}_{0} \in\left(0, \lambda_{c}\right)$. Then $K\left(\xi-\omega_{1}\right)<K(\xi)$, by choosing $\bar{\mu}_{0}<\frac{1}{\omega_{1}} \ln 2$. Therefore, it is necessary to choose that $0<\bar{\mu}_{0}<\min \left\{\mu_{c}, \lambda_{c}, \frac{1}{\omega_{1}} \ln 2\right\}$. Therefore, $K(\xi)$ is bounded as $\xi \rightarrow-\infty$ and $Q(\xi) \leq Q_{0} e^{\bar{\mu}_{0} \xi}$ for some positive number $Q_{0}$ and $\xi \in \mathbb{R}$. According to the definition of $Q(\xi)$, one has that

$$
\int_{-\infty}^{\xi} V(\eta) e^{-\bar{\mu}_{0} \xi} \mathrm{d} \eta<+\infty \text { and } \sup _{\xi \in \mathbb{R}}\left\{V(\xi) e^{-\bar{\mu}_{0} \xi}\right\}<+\infty,
$$

so $\int_{R} V(\xi) e^{-\mu \xi \mathrm{d} \xi}<+\infty$ for $\mu \in\left(0, \bar{\mu}_{0}\right)$. Moreover, it follows from $\left(A_{1}\right)-\left(A_{2}\right)$ that

$$
\begin{aligned}
& e^{-\bar{\mu}_{0} \xi} J * V(\xi)=\int_{-\infty}^{+\infty} J(y) e^{-\bar{\mu}_{0} y} V(\xi-y) e^{-\bar{\mu}_{0}(\xi-y)} \mathrm{d} y<+\infty, \\
& e^{-\bar{\mu}_{0} \xi} G * V(\xi)=\int_{0}^{T} \int_{-\infty}^{+\infty} G(y, s) e^{-\bar{\mu}_{0}(y+c s)} V(\xi-y-c s) e^{-\bar{\mu}_{0}(\xi-y-c s)} \mathrm{d} y \mathrm{~d} s<+\infty .
\end{aligned}
$$

In what follows, we define a smooth monotone auxiliary function $\eta(x) \in C^{\infty}(\mathbb{R},[0,1])$ by

$$
\eta(x)= \begin{cases}0, & x \in(-\infty,-2], \\ 1, & x \in[-1,+\infty),\end{cases}
$$

and let $\eta_{N}(x)=\eta\left(\frac{x}{N}\right)$. Multiplying the third equation of $(2.2)$ by $e^{-v \xi} \eta_{N}(\xi)$ and integrating the result on $\mathbb{R}$, we have

$$
\begin{aligned}
c \int_{\mathbb{R}} W^{\prime}(\xi) e^{-v \xi} \eta_{N}(\xi) \mathrm{d} \xi= & d \int_{\mathbb{R}}(J * W(\xi)-W(\xi)) e^{-v \xi} \eta_{N}(\xi) \mathrm{d} \xi+\beta \int_{\mathbb{R}} V(\xi) e^{-v \xi} \eta_{N}(\xi) \mathrm{d} \xi \\
& -\left(\gamma+\mu+\eta_{2}+\rho\right) \int_{\mathbb{R}} W(\xi) e^{-v \xi} \eta_{N}(\xi) \mathrm{d} \xi
\end{aligned}
$$

In addition,

$$
\begin{aligned}
& \int_{\mathbb{R}} J * W(\xi) e^{-v \xi} \eta_{N}(\xi) \mathrm{d} \xi \leq \int_{-\infty}^{+\infty} W(\xi) e^{-v \xi} \mathrm{d} \xi \int_{-\infty}^{+\infty} J(y) e^{-v y} \mathrm{~d} y \\
& \int_{\mathbb{R}} W^{\prime}(\xi) e^{-v \xi} \eta_{N}(\xi) \mathrm{d} \xi=v \int_{\mathbb{R}} W(\xi) e^{-v \xi} \eta_{N}(\xi) \mathrm{d} \xi-\int_{\mathbb{R}} W(\xi) e^{-v \xi} \eta_{N}^{\prime}(\xi) \mathrm{d} \xi .
\end{aligned}
$$

Substituting (4.11) and (4.12) into (4.10), one has that

$$
\begin{aligned}
& \left(c v+d+\gamma+\mu+\eta_{2}+\rho\right) \int_{\mathbb{R}} W(\xi) e^{-v \xi} \eta_{N}(\xi) \mathrm{d} \xi-c \int_{\mathbb{R}} W(\xi) e^{-v \xi} \eta_{N}^{\prime}(\xi) \mathrm{d} \xi \\
& -d \int_{-\infty}^{+\infty} J(y) e^{-v y} \mathrm{~d} y \int_{\mathbb{R}} W(\xi) e^{-v \xi} \mathrm{d} \xi \leq \beta \int_{\mathbb{R}} V(\xi) e^{-v \xi} \eta_{N}(\xi) \mathrm{d} \xi .
\end{aligned}
$$

By the definition of $\triangle_{2}(\lambda, c)$, it follows that $\triangle_{2}(v, c)=d \int_{-\infty}^{+\infty} J(y)\left(e^{-v y}-1\right) \mathrm{d} y-\gamma-\left(\mu+\eta_{2}+\right.$ $\rho)-c v<0$ for any $v \in\left(0, \lambda_{2}^{+}\right)$, that is, $c v+\gamma+\mu+\eta_{2}+\rho-d \int_{-\infty}^{+\infty} J(y)\left(e^{-v y}-1\right) \mathrm{d} y>0$. Hence, 
$\int_{\mathbb{R}} W(\xi) e^{-v \xi} \mathrm{d} \xi \leq \frac{\beta}{-\triangle_{2}(v, c)} \int_{\mathbb{R}} V(\xi) e^{-v \xi} \mathrm{d} \xi$, by taking $N \rightarrow+\infty$ on the both sides of (4.13) and combining the facts that $\lim _{N \rightarrow+\infty} \eta_{N}(\xi)=1$ and $\lim _{N \rightarrow+\infty} \eta_{N}^{\prime}(\xi)=0$. Therefore, $\int_{\mathbb{R}} W(\xi) e^{-v \xi} \mathrm{d} \xi<$ $+\infty$ for $v \in\left(0, \bar{\mu}_{0}\right)$. Furthermore, $V^{\prime}(\xi) e^{-\bar{\mu}_{0} \xi}<+\infty$ for any $\xi \in \mathbb{R}$, by the second equation of (2.2). Therefore, $\sup _{\xi \in \mathbb{R}}\left\{J * V(\xi) e^{-\bar{\mu}_{0} \xi}\right\}<+\infty, \sup _{\xi \in \mathbb{R}}\left\{G * V(\xi) e^{-\bar{\mu}_{0} \xi}\right\}<+\infty, \sup _{\xi \in \mathbb{R}}\left\{V^{\prime}(\xi) e^{-\bar{\mu}_{0} \xi}\right\}<$ $+\infty$, which leads to $\int_{-\infty}^{+\infty}\left[\alpha \frac{G * V(\xi)(G * V(\xi)+W(\xi))}{U(\xi)+G * V(\xi)+W(\xi)}-\gamma W(\xi)\right] e^{-\mu \xi} \mathrm{d} \xi<+\infty$, where $\mu \in\left(0, \bar{\mu}_{0}\right)$. From the second equation of $(2.2)$, we obtain

$$
\begin{aligned}
& d[J * V(\xi)-V(\xi)]-c V^{\prime}(\xi)+\alpha G * V(\xi)+\gamma W(\xi)-\left(\mu+\eta_{1}+\beta\right) V(\xi) \\
= & \alpha\left[G * V(\xi)-\frac{U(\xi) G * V(\xi)}{U(\xi)+G * V(\xi)+W(\xi)}\right] .
\end{aligned}
$$

Applying a two-sided Laplace transform on (4.14), one has that

$$
\triangle_{1}(\lambda, c) L(\lambda)=\int_{-\infty}^{+\infty}\left[\alpha \frac{G * V(\xi)(G * V(\xi)+W(\xi))}{U(\xi)+G * V(\xi)+W(\xi)}-\gamma W(\xi)\right] e^{-\lambda \xi} \mathrm{d} \xi,
$$

where $L(\lambda)=\int_{-\infty}^{+\infty} V(\xi) e^{-\lambda \xi} \mathrm{d} \xi$ for $0<\operatorname{Re} \lambda<\bar{\mu}_{0}$. Based on the properties of a two-sided Laplace transform, it follows that the domain on the right of (4.15) is the whole right half plane. By Lemma 2.1, we know that $\triangle_{1}(\lambda, c)>0$ for all $\lambda>0$ when $0<c<c_{*}$, and thus the domain of $L(\lambda)$ is $\lambda \in \mathbb{C}$ and $0<\operatorname{Re} \lambda<+\infty$. However, $\triangle_{1}(\lambda, c) \rightarrow+\infty$ as $\lambda \rightarrow+\infty$, owing to the definition of $\triangle_{1}(\lambda, c)$, which is a contradiction. Therefore, there are no bounded nontrivial positive solutions of (2.2) satisfying (2.3)-(2.4) for every $0<c<c_{*}$. This ends the proof.

\section{$5 \quad$ Numerical Simulation and Discussion}

Theorem 3.8, Theorem 3.11 and Theorem 4.1 provide a threshold condition for whether the disease spreads or not. Specifically, according to Theorems 3.8 and 3.11, if $c \geq c^{*}$ and $\left(A_{1}\right)-\left(A_{3}\right)$ hold, then there are traveling waves for (2.1). In addition, by Theorem 4.1 there are no traveling waves for $(2.1)$ when $0<c<c_{*}$ and $\left(A_{1}\right)-\left(A_{3}\right)$ hold. Furthermore, we show that the wave speeds $c^{*}$ and $c_{*}$ are influenced by diffusion and time delay. In fact, it follows from Lemma 3.1 that

$$
\begin{aligned}
& N_{-}\left(\lambda^{*}, c^{*}\right) \\
= & d \int_{-\infty}^{+\infty} J(y)\left(e^{-\lambda^{*} y}-1\right) \mathrm{d} y-c^{*} \lambda^{*} \\
& +\left\{\left[\alpha \int_{0}^{T} \int_{-\infty}^{+\infty} G(y, s) e^{-\lambda^{*}\left(y+c^{*} s\right)} \mathrm{d} y \mathrm{~d} s-\left(\mu+\eta_{1}+\beta\right)-\left(\gamma+\mu+\eta_{2}+\rho\right)\right]\right. \\
& +\sqrt{\left.\left[\alpha \int_{0}^{T} \int_{-\infty}^{+\infty} G(y, s) e^{-\lambda^{*}\left(y+c^{*} s\right)} \mathrm{d} y \mathrm{~d} s-\left(\mu+\eta_{1}+\beta\right)+\left(\gamma+\mu+\eta_{2}+\rho\right)\right]^{2}+4 \gamma \beta\right\} / 2} \\
= & 0,
\end{aligned}
$$

and

$$
\begin{aligned}
\triangle_{1}\left(\lambda_{*}, c_{*}\right)= & d \int_{-\infty}^{+\infty} J(y)\left(e^{-\lambda_{*} y}-1\right) \mathrm{d} y+\alpha \int_{0}^{T} \int_{-\infty}^{+\infty} G(y, s) e^{-\lambda_{*}\left(y+c_{*} s\right)} \mathrm{d} y \mathrm{~d} s \\
& -\left(\mu+\eta_{1}+\beta\right)-c_{*} \lambda_{*} \\
= & 0 .
\end{aligned}
$$


Furthermore, the derivative of $c^{*}\left(c_{*}\right)$ with respect to $d$ is greater than zero; that is, $\frac{\mathrm{d} c^{*}}{\mathrm{~d} d}>0$ and $\frac{\mathrm{d} c_{*}}{\mathrm{~d} d}>0$, which can be obtained by the implicit function theorem.

In order to obtain the effect of time delay on the wave speeds $c^{*}$ and $c_{*}$, the spatio-temporal delay term $G * V(x, t)=\int_{0}^{T} \int_{-\infty}^{+\infty} G(y, s) V(x-y, t-s) \mathrm{d} y \mathrm{~d} s$ turns into the space non-local term with time delay $\int_{-\infty}^{+\infty} G(y) V(x-y, t-\tau) \mathrm{d} y$ by taking $G(x, t)=\delta(t-\tau) h(x)$, therefore (5.1) and (5.2) become

$$
\begin{aligned}
N_{-}\left(\lambda^{*}, c^{*}\right)= & d \int_{-\infty}^{+\infty} J(y)\left(e^{-\lambda^{*} y}-1\right) \mathrm{d} y-c^{*} \lambda^{*} \\
& +\left\{\left[\alpha \int_{-\infty}^{+\infty} G(y) e^{-\lambda^{*}\left(y+c^{*} \tau\right)} \mathrm{d} y-\left(\mu+\eta_{1}+\beta\right)-\left(\gamma+\mu+\eta_{2}+\rho\right)\right]\right. \\
& \left.+\sqrt{\left[\alpha \int_{-\infty}^{+\infty} G(y) e^{-\lambda^{*}\left(y+c^{*} \tau\right)} \mathrm{d} y-\left(\mu+\eta_{1}+\beta\right)+\left(\gamma+\mu+\eta_{2}+\rho\right)\right]^{2}+4 \gamma \beta}\right\} / 2 \\
= & 0,
\end{aligned}
$$

and

$$
\begin{aligned}
\triangle_{1}\left(\lambda_{*}, c_{*}\right) & =d \int_{-\infty}^{+\infty} G(y) e^{-\lambda_{*}\left(y+c_{*} \tau\right)} \mathrm{d} y+\alpha \int_{-\infty}^{+\infty} G(y) e^{-\lambda_{*}\left(y+c_{*} \tau\right)} \mathrm{d} y-\left(\mu+\eta_{1}+\beta\right)-c_{*} \lambda_{*} \\
& =0 .
\end{aligned}
$$

Therefore, $\frac{d c^{*}}{d \tau}<0$ and $\frac{d c_{*}}{d \tau}<0$ can be verified by the implicit function theorem. Based on the above analysis, it can be seen that the longer delay $\tau$, the slower the wave speed $c^{*}\left(c_{*}\right)$, and the bigger the dispersal rate $d$, the faster the wave speed $c^{*}\left(c_{*}\right)$.

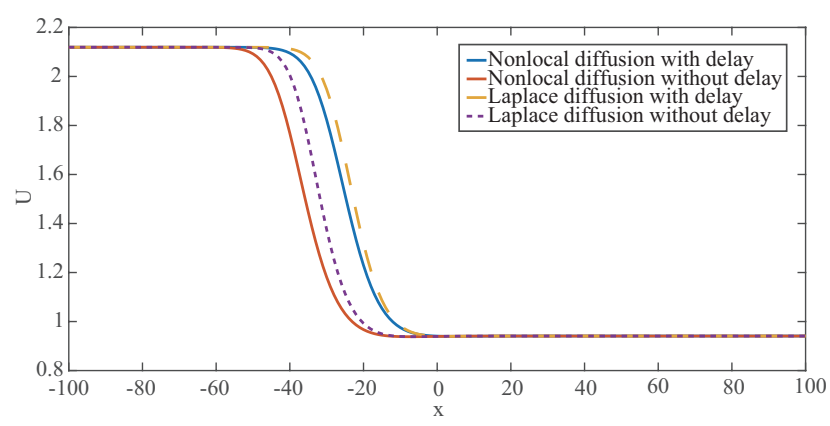

Figure 1 Traveling waves of $\mathrm{U}$

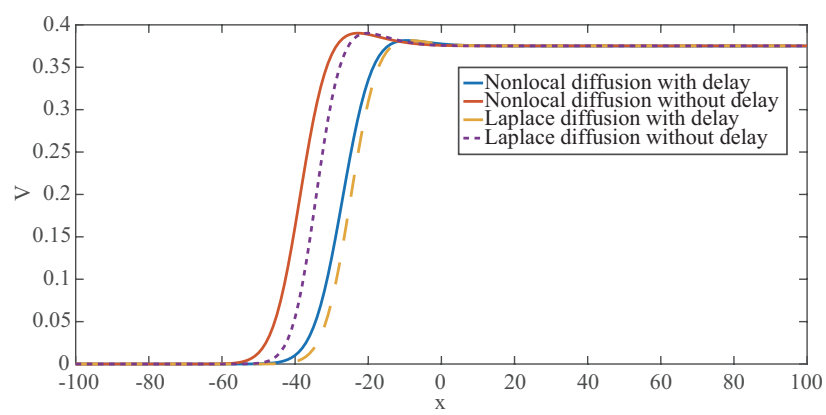

Figure 2 Traveling waves of $\mathrm{V}$ 


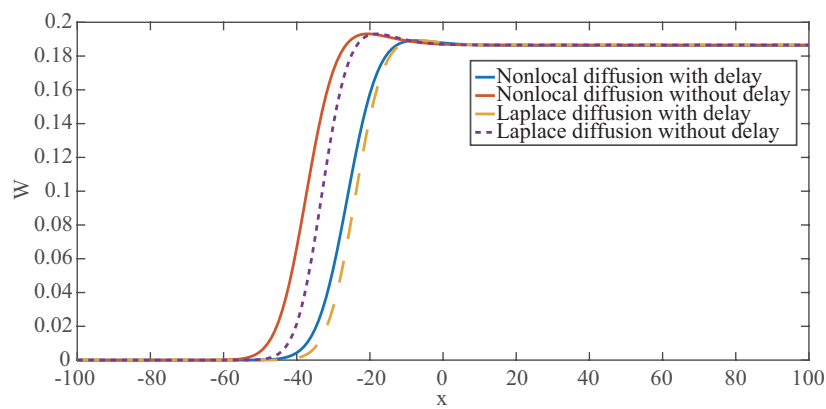

Figure 3 Traveling waves of $\mathrm{W}$

On the other hand, inspired by the work of Zhu et al. [12], the two-dimensional numerical simulations for traveling waves of an HIV/AIDS infection model with (or without) delay and local (or nonlocal) dispersal are demonstrated above (in view of the fact that the HIV/AIDS infection model is well-known, and as a kind of epidemic model with relapse).

In the process of numerical simulation, $d=0.1$, and the other correlation parameters are stated in the table below.

\begin{tabular}{c|cccccccc}
\hline Parameter & $\Lambda$ & $\alpha$ & $\gamma$ & $\beta$ & $\rho$ & $\mu$ & $\eta_{1}$ & $\eta_{2}$ \\
\hline Data & 0.338 & 0.8 & 0.5 & 0.4 & 0.0783 & 0.1595 & 0.19 & 0.0667 \\
\hline Data sources & \multicolumn{1}{c}{ References[12] } \\
\hline
\end{tabular}

In addition, $U_{0}=2.119122257, U^{*}=0.9407872997, V_{0}=0, V^{*}=0.3752146776, W_{0}=0$ and $W^{*}=0.1865579503$ can be obtained by a simple calculation.

As is shown in Figures 1-3, the spread of traveling waves can be accelerated by nonlocal dispersal, while it can be slowed down by time delay, that is, in biology, disease spread will be faster in the case of nonlocal dispersal and small time delay. This conforms with the above discussions in mathematical analysis.

Acknowledgements We would like to give thanks for the help in numerical simulations to doctoral student Mingzhen Xin of the School of Mathematics and Statistics at Lanzhou University.

\section{References}

[1] Aronson D G, Weinberger H F. Nonlinear diffusion in population genetics, combustion, and nerve pulse propagation. Partial Differential Equations and Related Topics, 1975, 446: 5-49

[2] Fife P C, Mcleod J B. The approach of solutions of nonlinear diffusion equations to traveling front solutions. Arch Rational Mech Anal, 1977, 65: 335-361

[3] Ducrot A, Magal P, Ruan S G. Travelling wave solutions in multigroup age-structured epidemic models. Arch Rational Mech Anal, 2010, 195(1): 311-331

[4] Wang X S, Wang H Y, Wu J H. Traveling waves of diffusive predator-prey systems: disease outbreak propagation. Discrete and Continuous Dynamical Systems, 2012, 32(9): 3303-3324

[5] Zhang T R, Wang W D, Wang K F. Minimal wave speed for a class of non-cooperative diffusion-reaction system. J Differential Equations, 2016, 260(3): 2763-2791

[6] Medlock J, Kot M. Spreading disease: integro-differential equtions old and new. Mathematical Biosciences, 2003, 184(2): 201-222 
[7] Coville J, Dupaigne L. Propagation speed of travelling fronts in nonlocal reaction-diffusion equations. Nonl Anal, 2005, 60(5): 797-819

[8] Yang F Y, Li Y, Li W T, Wang Z C. Traveling waves in a nonlocal dispersal Kermack-McKendrick epidemic model. Discrete and Continuous Dynamical Systems Series B, 2013, 18(7): 1969-1993

[9] Li W T, Yang F Y. Traveling waves for a nonlocal dispersal SIR model with standard incidence. Journal of Integral Equations and Applications, 2014, 26(2): 243-273

[10] Yang F Y, Li W T. Traveling waves in a nonlocal dispersal SIR model with critical wave speed. J Math Anal Appl, 2018, 458(2): 1131-1146

[11] Yang F Y, Li W T, Wang J B. Wave propagation for a class of non-local dispersal non-cooperative systems. Proceedings of the Royal Society of Edinburgh Section A Mathematics, 2019, 150(4): 1-33

[12] Zhu C C, Li W T, Yang F Y. Traveling waves in a nonlocal dispersal SIRH model with relapse. Computers and Mathematics with Applications, 2017, 73(8): 1707-1723

[13] Li Y, Li W T, Yang F Y. Traveling waves for a nonlocal dispersal SIR model with delay and external supplies. Appl Math Comput, 2014, 247(15): 723-740

[14] Zhang S P, Yang Y R, Zhou Y H. Traveling waves in a delayed SIR model with nonlocal dispersal and nonlinear incidence. J Math Phys, 2018, 59(1): 011513

[15] Yang Y, Yang Y R, Jiao X J. Traveling waves for a nonlocal dispersal SIR model equipped delay and generalized incidence. Electronic research archive, 2020, 28(1): 1-13

[16] Britton N F. Aggregation and the competitive exclusion principle. J Theoret Biol, 1989, 136(1): 57-66

[17] Britton N F. Spatial structures and periodic travelling waves in an integro-differential reaction-diffusion population model. SIAM J Appl Math, 1990, 50(6): 1663-1688

[18] Smith H L, Thieme H R. Strongly order preserving semiflows generated by functional differential equations. J Differential Equations, 1991, 93(2): 332-363

[19] Wang J B, Li W T, Yang F Y. Traveling waves in a nonlocal dispersal SIR model with nonlocal delayed transmission. Communications in Nonlinear Science and Numerical Simulation, 2015, 27(1/3): 136-152

[20] Wu S L, Ruan S G. Entire solutions for nonlocal dispersal equations with spatio-temporal delay: Monostable case. J Differential Equations, 2015, 258(7): 2435-2470

[21] Ma Z H, Yuan R. Traveling wave solutions of a nonlocal dispersal SIRS model with spatio-temporal delay. International Journal of Biomathematics, 2017, 10(5): 1-23

[22] Zhou X. Traveling wave solutions for nonlocal dispersal SIR models with spatio-temporal delays. Applied Mathematics and Mechanics, 2018, 39(5): 611-630

[23] Su S, Zhang G B. Traveling waves of a nonlocal dispersal equation with spatio-temporal delay. Journal of Lanzhou University of Arts and Science (Natural Sciences), 2020, 34(3): 9-13

[24] Yang Y R, Yang Y, Ma Z Y. Traveling waves for a nonlocal dispersal SIR model with renewal and spatio-temporal delay. Applicable Analysis, 2021. https://www.tandfonline.com/doi/full/10.1080/ 00036811.2021.1969369 